\title{
Influence of Phase Stability on the In Situ Growth Stresses in
}

\section{$\mathrm{Cu} / \mathrm{Nb}$ Multilayered Films}

\author{
Qianying Guo ${ }^{1}, \mathrm{Li} \mathrm{Wan}^{1}$, Xiao-xiang $\mathrm{Yu}^{1}$, Florian Vogel ${ }^{1}$ and Gregory B. Thompson ${ }^{1 *}$ \\ 1 The University of Alabama, Department of Metallurgical and Materials Engineering, \\ Tuscaloosa, AL 35487-0202, USA \\ *Corresponding author \\ E-mail address: gthompson@eng.ua.edu (Gregory B. Thompson)
}

Keywords: Thin film, multilayer, phase transformation, $\mathrm{Cu}, \mathrm{Nb}$, biphase diagram

\begin{abstract}
As the length scale of individual layers were reduced in a $\mathrm{Cu} / \mathrm{Nb}$ multilayer, a face centered cubic (fcc)-to-body centered cubic (bcc) and bcc-to-fcc transformations were noted for $\mathrm{Cu}$ and $\mathrm{Nb}$ respectively. In addition, at equal fractions of $\mathrm{Cu} / \mathrm{Nb}$ and at a very thin thickness, the layers vitrified. These phase transformations have been modeled using a thermodynamic phase diagram where strain, interfacial, and volumetric energy considerations were used to determine the phase as a function of the bilayer thickness and volume fraction in the multilayer. Using HRTEM, the evolution of the interface from semicoherent-to-coherent was observed as a function of phase and bilayer spacing and is discussed in terms of the interfacial energy contributions towards phase stability. The vitrification has been rationalized by the positive enthalpy of mixing with clustering quantified by atom probe tomography between these two species. All of these phase transformations have been related to real-time, intrinsic growth stress measurements. Upon phase transforming, a reduction in the compressive stress within all of the multilayers were noted. The stabilization of bcc $\mathrm{Cu}$ appeared to have a dramatic effect on the growth stresses of $\mathrm{Nb}$ that contributed to the compressive stress relaxation. This relaxation was captured in a molecular dynamics simulation of the multilayers and is contributed to the change in interfacial stress and stress distributions within the $\mathrm{Cu}$ layer. This demonstrates that phase
\end{abstract}


transformations provide a means to tailor film stress in nanolaminate materials.

\section{Introduction}

Multilayered thin films are widely applied in magnetic materials [1-3], high mechanical hardness coatings [4, 5], semiconductor and solar cell devices [6, 7], and radiation damage resistant surfaces [8-10]. Many of the applications listed above are generally based on the properties of specific crystal structures, chemical compositions, and stress states of the thin films that comprise the multilayers [11-26]. At finer nanometer length scales, these individual layers within the multilayer can undergo a phase transformation. For example, in the giant magnetoresistance $\mathrm{Co} / \mathrm{Cr}$ multilayers, Boher et al. reported a change from hexagonal close packed (hcp) to body centered cubic (bcc) Co when $1.5 \mathrm{~nm}$ thick Co layers were deposited between $198.5 \mathrm{~nm}$ thick Cr layers [27]. In contrast, Vavra et al. reported a bcc-to-hcp Cr phase transformation (not a Co transition) when the thickness of Co and Cr layers were $2 \mathrm{~nm}$ and $0.6 \mathrm{~nm}$ respectively [28]. Other metallic multilayers have also shown similar transformations in both layers. In $\mathrm{Cu} / \mathrm{Nb}$, Kung et al. [19] reported a transformation from face centered cubic (fcc) $\mathrm{Cu}$ to bcc $\mathrm{Cu}$ when Cu's layer thickness was less than $1.2 \mathrm{~nm}$ while others have reported a bcc-to-fcc $\mathrm{Nb}$ transformation in the same $\mathrm{Cu} / \mathrm{Nb}$ multilayers [29]. In the $\mathrm{Zr} / \mathrm{Nb}$ system, both hep-to-bcc $\mathrm{Zr}$ and bcc-to-hcp $\mathrm{Nb}$ transformations have also been reported [30, 31].

To explain such phase transformations, researchers have proposed both strain induced [16, 32, 33] and thermodynamic concepts [30, 31, 34, 35]. The former framework considers the change in phase to raise the energy of the crystal, but at a specific strain value, the lattice can relax to a lower energy, metastable phase as compared to the non-strained lattice phase. In the thermodynamic prospective, the transformed phase is considered to be in its lowest energy 
configuration based on a balance of interfacial and volumetric energy considerations. Using these two thermodynamic terms (i.e., volumetric and interfacial energies), Dregia et al. proposed a phase diagram, termed a biphase diagram, that could predict the phase change in multilayered thin films $[34,36]$ as a competition between these two terms and the size of the layer pairs. Here, the total Gibbs free energy change between the two phases, $\Delta G$, was normalized by the fixed surface area of the film and redefined as $\Delta g$. Any increase in the normalized volumetric energy must be off-set by the reduction in the interfacial energy, $\Delta \gamma$, between the two phases.

Using the Dregia et al. paper $[34,36]$ as a guide, we formulate its construction in terms of $\mathrm{Cu} / \mathrm{Nb}$ multilayers which will be the focus of this paper. The $\Delta g$ for any transformation is then written explicitly as

$$
\Delta g=2 \Delta \gamma+\Delta G_{N b} \mathrm{f}_{N b} \lambda+\Delta G_{C u}\left(1-\mathrm{f}_{N b}\right) \lambda
$$

where $\mathrm{f}_{N b}$ is the volume fraction of $\mathrm{Nb}, \lambda$ is the bilayer thickness or the sum of the individual thicknesses of a $\mathrm{Cu}$ and $\mathrm{Nb}$ layer pair, and $\Delta G_{i}$ is the volumetric free energy change per unit volume of $\mathrm{Cu}$ or $\mathrm{Nb}$. In this model, transformations in either layer can then be predicted dependent upon their thickness, which is simply the volume fraction of that element multiplied by $\lambda$. Once a layer is sufficiently thin, the change in interfacial energy reduction can become sufficient to offset the volumetric energy increase for such a transformation [30, 36].

From the prior reports for phase changes discussed above, we can now rationalize why different groups report different transformations even in the same multilayer type. For example, in the $\mathrm{Co} / \mathrm{Cr}$ system, each group explored a different region of the phase space that depended on the individual layer thickness (or volume fraction) within their bilayer. Using equation (1), regions of phase stability in $\mathrm{Cu}$ or $\mathrm{Nb}$ can then be mapped out as a function of the reported $\mathrm{f}_{N b}$ and the inverse bilayer thickness, $\lambda^{-1}[36]$. The development of such a $\mathrm{Cu} / \mathrm{Nb}$ phase map would 
be of particular interest to a larger scientific community as this system has been shown to exhibit excellent radiation damage control via their interfacial structure and number of interfaces, i.e. layer pairs [9]. A phase map would then assist in the engineering of the bilayer thicknesses where the optimal interfacial structure and phase is retained to ensure optimization of those and well as other properties.

To initiate our hypothetical (predicted) $\mathrm{Cu} / \mathrm{Nb}$ transformations, we have relied upon the transformation as reported by Kung et al. [19]. Here, a fcc-to-bcc $\mathrm{Cu}$ transformation occurred at $\mathrm{af}_{N b}=0.5$ and $\lambda=1.2 \mathrm{~nm}$ which has been plotted in Fig. 1. As noted above, others [29] have also reported a transformation in $\mathrm{Cu} / \mathrm{Nb}$ but their transformation was bcc-to-fcc $\mathrm{Nb}$ at the same volume fraction $\mathrm{f}_{N b}=0.5$ and at a larger bilayer thickness of $\lambda=20 \mathrm{~nm}$. Since most stabilized nanoscale transition in these types of multilayers have been in the one to few nanometer thickness range, we believe the Kung et al. report would provide the most reasonable initial guide. However, through this research, we will be able to verify if such a larger length scale transformation does indeed occur for $\mathrm{Nb}$.

The phase boundaries shown in Fig. 1 will have a slope given as

$$
\lambda^{-1} / \mathrm{f}_{N b}= \pm \Delta G_{N b} /(2 \Delta \gamma)
$$

based on equation (1). As the volume fraction of $\mathrm{Nb}$ decreases, the probability of a transformation in $\mathrm{Cu}$ would become less likely; as a result we have placed such transformations being divided by a dashed line in Fig. 1. In addition, strained $\mathrm{Cu}$ transformations are known to exist, such as the Bain transition [37], which would cause the phase boundary to be curved (not linear). Thompson et al. [30] have reported how equation (1) can be modified to account for such strain-based effects. Equation (3) is a reformulation of those effects for the $\mathrm{Cu} / \mathrm{Nb}$ multilayer. 


$$
\Delta g=2 \Delta \gamma+\left[\Delta G_{N b} \mathrm{f}_{N b}+\Delta G_{C u}\left(1-\mathrm{f}_{N b}\right)+Y m_{0}^{2}\right] \lambda
$$

where $Y$ is biaxial elastic modulus related with Young's modulus and Poisson's ratio of the film and $m_{0}$ is the natural misfit which equals the sum of strain (absolute value) in $\mathrm{Cu}$ layer and $\mathrm{Nb}$ layer at their interface [30]. However, the lack of additional reported transformations for either $\mathrm{Nb}$-rich or Nb-lean volume fractions prevents us to know the potential extent of such a curved boundary. Consequently, we will use the linear boundary as a first approximation.

Recent work by Wan et al. has shown the use of in situ stress measurements as a valuable technique in understanding the strain development in a thin film multilayer that is undergoing a phase transformation [38]. In that work, a series of equal layer thicknesses of $\mathrm{Ti} / \mathrm{Nb}$ were deposited and an initial tensile stress slope was noted to occur in Ti as it grew on $\mathrm{Nb}$ up to $\sim 2 \mathrm{~nm}$, whereupon it reverted to a compressive stress slope. This transition was found to take place at the bcc-to-hcp Ti phase transition. Even more interesting was that the stabilization of bcc $\mathrm{Ti}$ resulted in nearly 20 at.\% $\mathrm{Nb}$ intermixing within the Ti layer and contributed to a lowering of the volumetric free energy barrier for this phase transformation as $\mathrm{Nb}$ is a $\beta$-stabilizer in $\mathrm{Ti}$ [38].

In this new study, we propose to apply similar stress measurements to correlate the $\mathrm{Cu} / \mathrm{Nb}$ transformations to the stress evolution as the layers grow. We now extend this work to include non-equal layer thickness effects on the growth stress and what role, if any, do they have in tuning the overall multilayer stress. In such cases, the volume fraction effects may become more appreciable in the phase stabilization and related growth stresses. The $\mathrm{Cu} / \mathrm{Nb}$ system offers a distinctively different thermodynamic system than $\mathrm{Ti} / \mathrm{Nb}$ too. $\mathrm{Cu}$ and $\mathrm{Nb}$ are bulk immiscible species where intermixing would not be expected; in contrast, significant $\mathrm{Nb}$ was found to mix with the $\beta$-Ti phase. If mixing is eliminated, the possible phase changes would be very 
dependent on the interfacial energies. It is also worth to note that both fcc $\mathrm{Cu}$ and bcc $\mathrm{Nb}$ have relatively similar lattice constants $\left(\mathrm{a}_{\mathrm{Cu}}=0.362 \mathrm{~nm}\right.$ and $\left.\mathrm{a}_{\mathrm{Nb}}=0.331 \mathrm{~nm}\right)$. Through the biphase diagram coupled with the in situ stress measurements, we will then be able to understand when phase changes occur coupled with underlying diagnostic tools to characterize the multilayer response to the phase transformations to help reveal the mechanisms that contribute to nanoscale phase stability.

\section{Experimental and Computational Modeling Procedure}

A series of $\mathrm{Cu} / \mathrm{Nb}$ multilayers were sputter-deposited onto $\sim 270 \mu \mathrm{m} \mathrm{Si} \mathrm{[001]} \mathrm{substrates,}$ with each substrate having a native oxide on its surface, in an AJA ATC-1500 sputtering chamber. The total layer thickness for all films was $240 \mathrm{~nm}$ with bilayer thicknesses spanning 1 to $40 \mathrm{~nm}$. Sputter parameters were maintained to achieve three $\mathrm{Nb}$ layer volume fractions of $\mathrm{f}_{N b}$ $=0.25, \mathrm{f}_{N b}=0.5$ and $\mathrm{f}_{N b}=0.75$ in each respective bilayer multilayer. The pressure of the sputtering chamber was $<1.33 \times 10^{-6} \mathrm{~Pa}$ prior to sputtering and kept at $0.133 \mathrm{~Pa}$ during sputtering using ultra-high-purity argon as the working gas at a flow rate of $0.01 \mathrm{~L} / \mathrm{min}$. All multilayers were deposited at room temperature $\left(\sim 25^{\circ} \mathrm{C}\right)$.

The in situ growth stresses during deposition was recorded with a k-Space multi-beam optic sensor (MOS) [39]. The stresses in these films were then calculated based on the substrate curvature using the Stoney equation [40]:

$$
\sigma_{f}=\frac{E_{S}}{6\left(1-v_{S}\right)} \frac{t_{S}^{2}}{t_{f}}\left(\frac{1}{R}-\frac{1}{R_{0}}\right)
$$


where $\sigma_{f}$ is the film stress, $E_{s}$ is the Young's modulus of the substrate, $v_{s}$ is the Poisson's ratio for the substrate, $t_{s}$ is the substrate thickness, $t_{f}$ is the film thickness, and $1 / R_{0}$ and $1 / R$ are measured curvatures of the film before and during/after deposition, respectively.

Small angle X-ray reflectivity (XRR) and X-ray Diffraction (XRD) were conducted on a Philips X'pert diffractometer with a $\mathrm{Cu} \mathrm{K} \alpha$ source operated at $40 \mathrm{keV}$ and $30 \mathrm{~mA}$ [41]. XRR $\theta$ $2 \theta$ scans were undertaken to determine the layer thickness with the deposition rates determined by the layer thicknesses divided by the deposition run time. WinGIXA was used to analyze the obtained XRR data. XRD $\theta-2 \theta$ scans were applied for identification of phases and to determine the d-spacing in the growth direction of the layers. All scans were performed with an angular resolution of $0.005^{\circ}$ expect for the $\mathrm{Cu} / \mathrm{Nb} \mathrm{f}_{N b}=0.5, \lambda=1 \mathrm{~nm}$ sample, which had a resolution of $0.002^{\circ}$.

Plan-view transmission electron microscopy (TEM) samples were prepared by cutting a 3 mm disc with an ultrasonic cutter from the center of each wafer. First the back-side was ground with $\mathrm{SiC}$ paper to a thickness of $\sim 100 \mu \mathrm{m}$ and then the disc was dimpled to $<11 \mu \mathrm{m}$ using $6 \mu \mathrm{m}$ diamond paste as the abrasive. The dimpled samples were initially ion-milled at $4.0 \mathrm{keV}$ and finally polished at $1 \mathrm{keV}$ in a Gatan Precision Ion Polishing System (PIPS) using $\mathrm{Ar}^{+}$ions. Cross-section TEM samples were prepared by a focused ion beam (FIB) lift-out procedure [4245] in either a FEI Quanta 3D dual SEM-FIB or Tescan Lyra 3XM dual SEM-FIB. Initially milling was done at $30 \mathrm{keV}$ with step down ion currents from $1.0 \mathrm{nA}$ to $0.1 \mathrm{nA}$. A subsequent low $\mathrm{keV}$ clean-up at $5 \mathrm{keV}$ at a beam current of $50 \mathrm{pA}$ was done to remove the surface damage created by the $\mathrm{Ga}^{+}$implantation into the sidewalls of the foils [42]. The foils were characterized using a FEI Tecnai G ${ }^{2}$ F20 Super-twin (Scanning) Transmission Electron Microscope ((S)TEM) operated at $200 \mathrm{keV}$. 
Atom probe tomography (APT) samples were prepared using a similar FIB lift-out technique. Annular milling patterns of increasingly smaller inner and outer diameters were used to sharpen each tip [42-44]. The APT was conducted in a LEAP 5000 XS (Cameca Scientific Instruments). Thermally induced field evaporation was performed with picosecond pulses from a green laser $\left(\lambda=355 \mathrm{~nm}, 250 \mathrm{kHz}, 20\right.$ to $\left.40 \mathrm{pJ} \cdot \mathrm{pulse}^{-1}\right)$. The experimental parameters were controlled to maintain a constant evaporation rate of 0.005 ions per pulse. For all measurements, the temperature of the sample was kept between 30-40 K. The 3D-data sets were reconstructed using the IVAS 3.3.12 software package (Cameca Scientific Instruments) following the procedures outlined in Ref. [46].

To better elucidate the links between the structural phase stability with the residual stress evolution, a molecular dynamic (MD) simulation was undertaken utilizing the LAMMPS code [47]. The $\mathrm{Cu}$ and $\mathrm{Nb}$ embedded atom model potentials used can be found in reference [9]. The in-plane $\mathrm{x}-\mathrm{y}$ directions for the thin film model was periodic with the growth $\mathrm{z}$ direction separated by a vacuum region. Alternative $\mathrm{Cu} / \mathrm{Nb}$ single crystal multilayers with different crystalline structures and the Kurdjumov-Sachs orientation relationship was used for bulk phase stability and an equivalent, coherent interface of the closest packed planes used for the phase stabilized conditions - both orientations of which matched experiments. For the $1 \mathrm{~nm} / 1 \mathrm{~nm}(\mathrm{bcc} \mathrm{Cu} / \mathrm{bcc} \mathrm{Nb}$ ) multilayers, a $9 \mathrm{~nm} \times 9 \mathrm{~nm} \times 56 \mathrm{~nm}$ simulation cell was used with the bottom two layers of $\mathrm{Nb}$ fixed to prevent movement. For the $2 \mathrm{~nm} / 2 \mathrm{~nm}$ and $20 \mathrm{~nm} / 20 \mathrm{~nm}$ fcc $\mathrm{Cu} / \mathrm{bcc} \mathrm{Nb}$ multilayers, a $5.7 \mathrm{~nm} \times 6.5 \mathrm{~nm} \times 400 \mathrm{~nm}$ simulation cell was used. The velocity Verlet algorithm and NoséHoover thermostat [48-51] were employed to solve the motion equations for the atoms and control the system temperature at $300 \mathrm{~K}$. After $1000 \mathrm{ps}$, the structures were fully relaxed to their equilibrium configurations. The outputs were then visualized by OVITO software [52] and the 
phases were distinguished by the common neighbor analysis (CNA) method [53]. This method of simulation was previously used and successfully demonstrated by the authors for $\mathrm{Ti} / \mathrm{Nb}[38]$.

\section{Experimental Results}

\subsection{Phase identification and microstructure}

\subsubsection{X-ray diffraction}

Fig. 2 shows the results of $\theta-2 \theta$ XRD scans for $\mathrm{Cu} / \mathrm{Nb}$ multilayers with varying bilayer thicknesses and $\mathrm{Nb}$ volume fractions, $\mathrm{f}_{N b}$. Fig. 2(a)'s XRD scans for the $\mathrm{f}_{N b}=0.25 \mathrm{Cu} / \mathrm{Nb}$ multilayers indicated peak shifts and peak merging with a continual reduction of the bilayer spacing. For a bilayer thickness of $\lambda=40 \mathrm{~nm}$, individual phases from each layer type can be

clearly indexed to their equilibrium phases. At $\lambda=4 \mathrm{~nm}$, a single peak about $41.28^{\circ}$ is noted with satellite reflections on either side. Satellite peaks are a result of constructive interference created by the repeating supercell structure of the multilayer stack $[54,55]$. As the bilayer thickness is gradually reduced to $2 \mathrm{~nm}$ to $1.2 \mathrm{~nm}$, this main peak exhibited a modest change in its $2 \theta$ location with a shift spanning 0.1 to $0.3^{\circ}$. In addition, the satellite peaks separated further from the major peak consistent to the decreasing bilayer spacing.

Similar XRD patterns of $\mathrm{f}_{N b}=0.5$ and 0.75 multilayers are shown in Fig. 2(b) and (c). In Fig. 2(b) and (c), at $\lambda=40 \mathrm{~nm}$, both types of multilayers revealed the bulk stable phases. When the bilayer thickness decreased to $8 \mathrm{~nm}$ for both of these $\mathrm{Nb}$ volume fractions, the $\mathrm{Nb}$ and $\mathrm{Cu}$ peaks shifted in comparison to their prior $\lambda=40 \mathrm{~nm}$ positions with the additional diffraction associated with satellite reflections. With $\lambda=4 \mathrm{~nm}$, a single peak formed for each of these volume fractions with corresponding satellite peaks on either side of this single peak. For the $\mathrm{f}_{N b}$ 
$=0.5$ at $\lambda=1 \mathrm{~nm}$, the single peak is very board (reminiscence of an amorphous reflection) as compared to the other peaks for equivalent bilayer spacings.

With the presence of a single peak, as well as satellite reflections, for each of these volume fractions, phase identification can be difficult requiring a complimentary diffraction technique to clearly discern the phase.

\subsubsection{Electron diffraction}

To further assist in the phase identification, selected area electron diffraction (SAED) was employed. Fig. 3 are representative plan-view diffraction patterns for the $\mathrm{f}_{N b}=0.25$ multilayers. Fig. 3(a) confirmed that the $30 \mathrm{~nm} \mathrm{Cu} / 10 \mathrm{~nm} \mathrm{Nb}$ multilayer to be fcc $\mathrm{Cu}$ and bcc $\mathrm{Nb}$ phases. In particular, clear fcc and bcc rings are individually indexed. When the bilayer thickness was reduced to $4 \mathrm{~nm}$, Fig. 3(b), the bulk phases are still present. For $1.5 \mathrm{~nm} \mathrm{Cu} / 0.5 \mathrm{~nm}$ $\mathrm{Nb}$ multilayer, Fig. 3(c), clear distinction between the fcc and bcc rings became more difficult to ascertain with the first inner ring being board in its intensity. This could suggest that the $\mathrm{Nb}$ has begun to undergo a bcc-to-fcc phase transformation (or at least very strained). However, we were able to observe a very faint bcc $\{200\}$ ring suggesting that the $\mathrm{Nb}$ has not fully transformed. At $\lambda$ $=1.2 \mathrm{~nm}$, the multilayer can be continuously indexed as a fcc phase. It is worth to note that hydrogen can stabilized an fcc $\delta$ - $\mathrm{NbH}$ phase; however, we do not believe this transition to be this phase because the lattice parameter $\mathrm{a}_{\delta-\mathrm{NbH}}=0.455 \mathrm{~nm}[56]$ whereas the fcc $\mathrm{Nb}$ phase above has an $\mathrm{a}_{\mathrm{fcc}-\mathrm{Nb}} \approx 0.36 \mathrm{~nm}$. Furthermore, plan-view foils, in comparison to cross-sectional foils, have been shown to be less susceptible to hydrogen infiltration and artifact stabilization effects [ 34 , 57].

Fig. 4 showed the representative plan view diffraction patterns at $\mathrm{f}_{N b}=0.5$. Consistent with the XRD results, when the bilayer thickness equaled to $40 \mathrm{~nm}$ and $4 \mathrm{~nm}$, both layers are in 
their bulk equilibrium structures with fcc $\mathrm{Cu}$ and bcc $\mathrm{Nb}$ rings indexed, Fig. 4(a) and (b) respectively. As the bilayer thickness decreased to $2 \mathrm{~nm}$ the rings can be consistently indexed to a bcc structure. When the bilayer became $1 \mathrm{~nm}$, the film did not show clear, multiple reflections as before but a very diffuse inner ring that is consistent with an amorphous structure. This diffraction pattern in Fig. 4(d) was consistent with the very board single peak noted in the XRD scan, Fig. 2(b). This suggested that a second phase transformation has occurred with decreasing bilayer thickness where the multilayer has now vitrified.

The selected plan view diffraction patterns at $\mathrm{f}_{N b}=0.75$ are shown in Fig. 5. Similar to the $\mathrm{f}_{N b}=0.25$ and 0.5 diffraction patterns, both fcc rings and bcc rings are present when the bilayer thickness equaled $40 \mathrm{~nm}$ and $4 \mathrm{~nm}$. As the bilayer thickness decreased to $2 \mathrm{~nm}$ and 1.2 $\mathrm{nm}$, the diffraction rings were indexed to a bcc phase. Unlike Fig. 4(c) and (d), all the rings (especially the inner most ring) in Fig. 5(c) and (d) are relatively sharp and any evidence vitrification diffraction was not found. Copper also has a hydride phase, but it is the wurtzite crystal structure with distinct a- and c- lattice parameters [58] whose reflections were not observed in the plan-view diffraction pattern. As with fcc $\mathrm{Nb}$, the bcc $\mathrm{Cu}$ is stabilized by the size and energy dependent effects.

\subsubsection{Film microstructure}

As thin film stress is a function of the microstructural evolution, we have quantified the average grain size (determined by plan view precession electron diffraction) as well as qualitative imaging that confirmed the presence of columnar grains within the multilayers. The average grain sizes spanned approximately $38 \mathrm{~nm}$ to $45 \mathrm{~nm}$ regardless of volume fraction or bilayer spacing. This suggests that the grain size is relatively invariant across the films and will have a less significant effect on stress as compared to other factors, such as bilayer spacing, 
volume fraction of material, and phase. Representative grain size histograms for the films can be found in the appendix as Fig. A1. A columnar grain morphology was observed in each of the multilayers, with representative cross-sectional images shown in Fig. 6. When the bilayer thickness was sufficiently thin, the columnar morphology spanned through the layer pairs in contrast to the very distinct columnar grains within the layers for the thicker layers - compare Fig. 6(a) to 6(b)-(c).

\subsection{In situ growth stress evolution}

With the phase stability of each multilayer established, the stress evolution during their growth is now reported. Thin film stress is intimately linked to the microstructure of the film, including grain size and grain morphology. However, as reported above, these films exhibited relatively similar grain sizes and were found to have a columnar grain morphology. This rather consistent microstructure between the multilayers is fortunate in that changes in stress changes can then be more directly established with the volume fraction of the multilayer, the bilayer spacing, and even phase stability [59]. Fig. 7(a), (b) and (c) are plots of the stress-film thickness product vs. film thickness for each of these multilayers at each of the volume fractions with Fig. 7(d)-(i) being selected regions of the scans to reveal representative growth stresses for individual layers at different volume fractions and bilayers. For all the multilayers, when they are in the post-coalescence state [60], the stress-thickness product had an overall compressive behavior, with the films becoming ever more compressive with $\mathrm{Nb}$ content and bilayer thickness until a critical bilayer spacing was achieved and the stress relaxed. The modulated stress within each line (see inset image in Fig. 7(b)), corresponded to the growth of each individual layer on the other in the multilayer stack; these modulations are also discerned more clearly in Figs. 7(d)-(i). 
Similar types of modulated growth for $\mathrm{Cu}$ on other immiscible metal surfaces have been reported for $\mathrm{Cu} / \mathrm{Ag}$ multilayers [61].

In Fig. 7(a), $\mathrm{f}_{N b}=0.25$, the compressive stress increased from $\lambda=40 \mathrm{~nm}$ to $2 \mathrm{~nm}$, after which the slopes of the curves tended to gradually recover to a reduced compressive state. The stress modulations between the layer-pairs became less and less pronounced with decreasing bilayer spacing, which may be due to the sensitivity of the scan to capture such thin layers. Atom probe tomography, to be discussed below, revealed that a chemical modulation was still present for these very thin Nb-lean (as well as forthcoming Nb-rich compositions). For the $\lambda=40 \mathrm{~nm}$ multilayer, groupings of approximately 6 layer pairs showed a sinusoidal stress response within each grouping as well as each grouping having a distinct increase in its compressive stress between each group. This film was grown an additional two times and the same grouping stress response was noted.

For Fig. $7(\mathrm{~b}), \mathrm{f}_{N b}=0.5$, as $\lambda$ decreased from $40 \mathrm{~nm}$ to $4 \mathrm{~nm}$, the total film increased its compressive stress. This compressive stress change was much larger than the $\mathrm{f}_{N b}=0.25$. Upon plotting the individual layer growth, Fig. 7(e) and (h), the $\mathrm{Cu}$ film stress gradient with thickness is relatively invariant but the growth of $\mathrm{Nb}$ resulted in a significant compressive stress drop between each $\mathrm{Cu}$ layer which drove the entire multilayer towards an ever increasing compressive stress state with decreasing bilayer thickness until $\lambda=4 \mathrm{~nm}$. At bilayers less than $4 \mathrm{~nm}$, the compressive stress relaxed in these $\mathrm{Cu} / \mathrm{Nb}$ multilayers. The surface energy of $\mathrm{Nb}$ is nearly twice that of $\mathrm{Cu}$ [62]; this stark difference would contribute to adatom mobility variations and their corresponding differences in growth stress responses between the two layers. Labat et al. [59] reported something similar in that the stress state was near zero for a Au layer grown on Ni layer because of the formation of an out-of equilibrium solid solution at the interface that expanded the 
lattice parameter. In our work, it was not necessarily a solid solution but rather a fcc-to-bcc $\mathrm{Nb}$ phase change (confirmed at $\lambda=2 \mathrm{~nm}$ ) that resulted in the change in the layer's growth stress.

Finally, Fig. 7(c), $\mathrm{f}_{N b}=0.75$, revealed an ever increasing compressive stresses which is contributed to the high volume fraction of $\mathrm{Nb}$ layer thicknesses in the film, Fig. 7(f). This compressive stress increased from $\lambda=40 \mathrm{~nm}$ to $2 \mathrm{~nm}$, which was similar to the other two volume fractions. With bilayer thickness of $1.2 \mathrm{~nm}$, the compressive stress relaxed but unlike the prior to multilayers, at this thinnest $1.2 \mathrm{~nm}$ bilayer, the stress state was still relatively more compressive than the 'bulk' $\lambda=40 \mathrm{~nm}$ condition. In Fig. 7(i), the fcc-to-bcc Cu phase changed at $\lambda=2 \mathrm{~nm}$ and again did not appear to alter the Cu's growth stress gradient with thickness (i.e., it remained relatively invariant) but, like $\mathrm{f}_{N b}=0.5$, the $\mathrm{Nb}$ growth stress gradient with thickness became less compressive.

\subsection{Interfacial structure evolution}

Fig. 8 shows the high resolution TEM (HRTEM) images for the $\mathrm{f}_{N b}=0.25$ multilayers. The dominant orientation relationship of the $\mathrm{fcc} / \mathrm{bcc}$ interfaces in $\mathrm{Cu} / \mathrm{Nb}$ multilayers is Kurdjumov-Sachs (KS) relationship, i.e., (111) $)_{\mathrm{fcc}} / /(110)_{\mathrm{bcc}},[110]_{\mathrm{fcc}} / /[111]_{\mathrm{bcc}}$, as reported in references [63-65]. In Fig. 8(a), $\mathrm{f}_{N b}=0.25$ and $\lambda=40 \mathrm{~nm}$, misfit dislocations can be observed in the $\mathrm{Nb}$ layer (comparable to prior reports in Ref.[66]). The presence of these misfit dislocations indicated that this interface is semi-coherent. Similar bulk phases of $\mathrm{Cu} / \mathrm{Nb}$ are seen for the $\mathrm{f}_{N b}=$ 0.25 at $\lambda=4 \mathrm{~nm}$. Each of these cross-sections' phases are consistent with their plan-view diffraction in Fig. 3. However, for the $\lambda=4 \mathrm{~nm}$ in Fig. 8(b), the HRTEM image did not reveal misfit dislocations at the interface for the field of view imaged but rather a modest distortion of the lattice planes at the interface suggesting a coherent interface. A slight shift in the XRD pattern, Fig. 2, was also noted for this particular multilayer. As the bilayer thickness reduced to 2 
nm, Fig. 8(c), portions of the interface stayed similarly distorted as noted by the red lines as a guide to the eye in tracing the lattice fringes across the interface. Other regions within this crosssection along the interface appeared to be relatively distortion free (continuous and unbent lattice fringes indicated by the guide to the eye green lines). Wang et al. [64] reported a molecular dynamics simulation $\mathrm{Cu} / \mathrm{Nb}$ multilayers where relatively distortion free bcc $\mathrm{Cu}$ regions were surrounded by Vernier misfits. Though we did not observe clear dislocations in our images, the presence of distorted and relatively distortion free bcc $\mathrm{Cu}$ is suggestive that our experimental findings may confirm the prior simulation predictions.

Finally, for the $\mathrm{f}_{N b}=0.25$ at $\lambda=1.2 \mathrm{~nm}$, the multilayer revealed a high proportion of continuous and unbent lattice fringes suggesting a loss of coherent distortion across the interface, Fig. 8(d). However, we do note that when it comes to a very thin bilayer thickness $(\sim 1 \mathrm{~nm})$, it is difficult to distinguish the $\mathrm{Cu}$ layer from the $\mathrm{Nb}$ layer by the contrast in the HRTEM images. Here we noted $\sim 1 \mathrm{~nm}$ bright and dark 'clusters' within the film and across the interfaces. These clusters will be further elaborated upon with the atom probe results below.

\subsection{Chemical analysis across interfaces}

Fig. 9 is the atom probe tomography chemical modulation analysis across the interfaces for the $\lambda=2 \mathrm{~nm} / \mathrm{f}_{N b}=0.5 ; \lambda=1.2 \mathrm{~nm} / \mathrm{f}_{N b}=0.25 ; \lambda=1.2 \mathrm{~nm} / \mathrm{f}_{N b}=0.75 ;$ and $\lambda=1 \mathrm{~nm} / \mathrm{f}_{N b}=0.5$. The time of flight mass spectrum only detected the metallic species and a small hydrogen gas peak, which is a known vacuum contaminate. No argon gas incorporation or oxygen was found to be in the films. As described in section 3.1.2, the hydrogen presence is not believed to have created artifacts in the phase stabilization evident by not observing the hydride lattice parameter for niobium or lower symmetry crystal structure for copper hydride. A representative mass spectrum can be seen in the appendix, as Fig. A2. 
Either a $1 \mathrm{D}$ concentration profile or spatial distribution map was taken normal to the layered interfaces for these measurements for each of these bilayers. For the $\lambda=2 \mathrm{~nm} / \mathrm{f}_{N b}=0.5$ multilayer, Fig. 9(a), a chemically modulated structure was found with a high proportion of intermixing of $\sim 48 \% \mathrm{Nb}$ atoms in each of the $\mathrm{Cu}$ layers with $\sim 46 \% \mathrm{Cu}$ atoms in each of the $\mathrm{Nb}$ layers. Though these species are 'bulk' immiscible, the atom probe results revealed mixing at the nanoscale. Coffey and Barmak [67] have previously noted that interfacial energies can have a significant impact on the driving forces that promote intermixing in thin film multilayers. At $\lambda=$ $1.2 \mathrm{~nm}$ for either $\mathrm{f}_{N b}=0.25$ or $\mathrm{f}_{N b}=0.75$, a layered chemical modulation is still present, proven by $\mathrm{Cu}$ spatial distribution maps; however, the layers themselves are again highly intermixed, Fig. 9(b) and (c). Finally, at $\lambda=1 \mathrm{~nm} / \mathrm{f}_{N b}=0.5$, the atom map revealed a loss of a layered chemical modulations and clear $\mathrm{Cu}$ and $\mathrm{Nb}$ clustering at the remnants of the prior layered interfaces, Fig. 9(d) [26]. Such clustering likely explained the similarly sized contrast seen in the HRTEM image in Fig. 8(d). This level of intermixing at these length scales have also been quantified in a series of de-vitrificated $\mathrm{Cu}-\mathrm{Nb}$ alloy films [68-70].

\section{Discussion}

From the phase identification, as determined by X-ray and electron diffraction, for each set of multilayers with different $\mathrm{f}_{N b}$, the phase diagram of Fig. 1 is re-plotted as Fig. 10. This more holistic phase diagram confirmed the prior predicted phase diagram regions but with some notable improvements. First, the additional multilayers grown in this study have more clearly delineated the phase space as well as identified a new amorphous phase regime for the thinnest bilayer studied at $\mathrm{f}_{N b}=0.5$. Interestingly, this amorphous region is not present on either $\mathrm{Cu}$-rich or $\mathrm{Nb}$-rich sides of the diagram suggesting that the phase field is limited near equal compositions at these very thin thicknesses. In addition, the boundaries between the crystalline phases in the 
equal and $\mathrm{Nb}$-rich volume fractions are curved, not linear, indicting the effects of strain in stabilizing the bcc $\mathrm{Cu}$ phase. Finally, the prior predicted $\mathrm{fcc} \mathrm{Nb}$ phase in $\mathrm{Cu} / \mathrm{Nb}$ discussed in the introduction [29] is significantly shifted to a much lower bilayer spacing value and is in the $\mathrm{Nb}$ lean region with. We did not confirm this transformation at $\mathrm{f}_{N b}=0.5$. Our work agrees with the report by Kung et al. [19] and is more consistent with prior phase transformations seen in other similar multilayered structures $[19,30,38]$. We suspect that the prior, larger layer thickness stabilization of fcc $\mathrm{Nb}$ reported by Zhang et al [29] is a result of the aforementioned fcc $\mathrm{NbH}$ artifact effect. In their paper, they report their fcc lattice parameter to be $\approx 0.4233 \mathrm{~nm}$, which can be easily rationalized as a strained hydride.

Using the slope of these experimentally determined boundaries, the change in interfacial energy, $\Delta \gamma$, between the crystalline phases was ascertained using equations (1)-(3). The slope of the fcc $\mathrm{Cu} / \mathrm{bcc} \mathrm{Nb}$ and $\mathrm{fcc} \mathrm{Cu} / \mathrm{fcc} \mathrm{Nb}$ phase boundary (Nb-lean volume fraction) in Fig. 10 is approximately 1.48. Based on the atom probe results, the intermixing of $\mathrm{Cu}$ into $\mathrm{Nb}$ will lower the $\Delta G_{N b}$ from $\approx 1.29 \times 10^{9} \mathrm{Jm}^{-3}(\mathrm{Nb}-0 \mathrm{Cu})[71]$ to $\approx 0.83 \times 10^{9} \mathrm{Jm}^{-3}(\mathrm{Nb}-46 \mathrm{Cu})$, with the mixed volumetric energies calculated from CALPHAD [71]. Using equation (2), the $\Delta \gamma_{\mathrm{f}_{\mathrm{v} b}}=0.25 \approx-280$ $\mathrm{mJm}^{-2}$ for a bcc-to-fcc $\mathrm{Nb}$ transformation. The linear boundary also suggested that coherency strains were not significant in driving the transformation. Those insights can also be gleaned from the HRTEM micrographs of Fig. 8. As the Nb-lean layers reduced their bilayer thickness, the fcc $\mathrm{Cu} / \mathrm{bcc} \mathrm{Nb}$ went from a semi-coherent interface to a coherent interface while still retaining their bulk phase structures. Upon further reduction, the coherent fcc $\mathrm{Cu} / \mathrm{fcc} \mathrm{Nb}$ phase formed and continued to retain the prior coherent interface.

In the equal thickness and $\mathrm{Nb}$-rich regime of the phase diagram, the phase boundary between fcc $\mathrm{Cu} / \mathrm{bcc} \mathrm{Nb}$ and bcc $\mathrm{Cu} / \mathrm{bcc} \mathrm{Nb}$ fields is significantly curved. Using equation (3), 
coupled with the APT results for the extent of $\mathrm{Nb}$ intermixing with $\mathrm{Cu}$, the $\Delta G_{C u}$ was reduced from $5.14 \times 10^{8} \mathrm{Jm}^{-3}(\mathrm{Cu}-0 \mathrm{Nb})$ to $\approx 2.20 \times 10^{8} \mathrm{Jm}^{-3}(\mathrm{Cu}-48 \mathrm{Nb})$ and the interfacial energy reduction being $\approx-430 \mathrm{mJm}^{-2}$ for this fcc-to-bcc transformation.

Though we have been able to ascertain the crystallographic phase transformations through a reduction of interfacial energy, the vitrification of both phases for the thinnest bilayer at the equal layer thicknesses offers a new wrinkle in the transformation behavior in this multilayer system. The APT results for this multilayer, Fig. 9(d), revealed remnants of a layered structure which were now broken up as clusters of $\mathrm{Nb}$ and $\mathrm{Cu}$. The lack of a continuous layer at this targeted bilayer is believed to be a result of the large surface energy differences between $\mathrm{Cu}$ and $\mathrm{Nb}$, which as mentioned above is a factor of two. Since prior multilayers that were able to be intermixed but retained a crystalline phase (Fig. 9) suggested that the stabilization of the glassy phase for $\mathrm{f}_{N b}=0.5$ appeared more to do with the breakdown of the interfaces themselves in the multilayers. This is particularly interesting result as in prior reports in other multilayers where the presence of a multilayered interface can actually stabilize the metallic glass phase in very thin layers [72]. Here, the interfaces are no longer laminate but those created by the intrinsic clustering of $\mathrm{Cu}$ and $\mathrm{Nb}$. When the films were either $\mathrm{Cu}$ or $\mathrm{Nb}$ rich in volume fraction, the layered interfaces are not as sensitive to this break down and the film, even intermixed, retained the crystalline phase. Thus the vitrification space is composition dependent, even at small bilayers. Banerjee et al. [73] have co-sputtered alloys of varying $\mathrm{Cu}-\mathrm{Nb}$ amounts and noted that the glassy phase formed with the $\mathrm{Nb}$ content varied between approximately 30 to 70 at.\% $\mathrm{Nb}$. Our 'mixed' multilayered results would be consistent with their alloy film findings.

With the phases stability established, we now address the consequence of this behavior on the multilayers' growth stress. In general, as the bilayer thicknesses for all three $\mathrm{f}_{N b}$ changed, 
the multilayers became more compressive. By plotting the overall growth stress, taken as a single linear fit to the growth curves in Figs. 7(a)-(c), versus the bilayer thickness, the stress relaxation at specific bilayers becomes readily apparent and can be easily compared across the multilayers, Fig. 11.

First, with increasing $\mathrm{Nb}$ volume fractions within the multilayers, the compressive stress increased. At a specific bilayer thickness, each of these multilayers experienced a stress relaxation with the $\mathrm{f}_{N b}=0.5$ and 0.75 films occurred at $\lambda=2 \mathrm{~nm}$. For these volume fractions, the bilayer spacing, $\lambda=2 \mathrm{~nm}$, is also the thickness where the fcc-to-bcc $\mathrm{Cu}$ transition occurred and would be amenable to a change in interfacial stress between the two layers. We noted that this interface was also coherent, Fig. 12. Labar et al. [59] reported a constituent equation that can estimate the growth stress per unit length of a single layer in terms of this interfacial stress. Using this equation as a guide, we have expanded it to our multilayered system as follows:

$$
F=\sigma h+n\left(f_{C u-N b}+f_{N b-C u}\right)
$$

where $F$ is the overall growth stress per unit length, $\sigma$ is volumetric stress which equals the biaxial modulus of the $\mathrm{Cu} / \mathrm{Nb}$ multilayers multiplied by the misfit at the interfaces, $h$ is the total thickness of the multilayer, $n$ is the number of bilayer pairs which equals $h$ divided by $\lambda$, and $f_{A-B}$ is the A-B interfacial stress. The biaxial stress was computed from the weighted averages of $\mathrm{Nb}$ content given from the computed biaxial stress at $\mathrm{f}_{N b}=0.5$ reported in reference [74]. The average interfacial stress values were measured from the in situ growth stress measurements recorded during deposition that followed the procedure reported by $\mathrm{Li}$ et al. $[38,75]$. These collective values, with equation (5), then predicted what the overall growth stress per unit length would be for each multilayer, Table 1. 
The calculated results shown in Table 1 exhibited a similar trend with the experimental data shown in Fig. 7(b), which was with decreasing bilayer thickness from $40 \mathrm{~nm}$ to $4 \mathrm{~nm}$, the compressive growth stress increased. A continual reduction of the bilayer thickness resulted in an exhibited stress relaxation. The computed overall growth stress values and their corresponding experimental values for each multilayer, Table 1 , were in relatively close agreement except for the $\mathrm{f}_{N b}=0.5$ at $\lambda=4 \mathrm{~nm}$. This particular film retained the bulk phases just prior to transformation of the $\mathrm{Cu}$ layer. This film state was significantly strained which could have altered the biaxial stress value taken from the computed (or idealized) condition referenced in [74]. Note that the computed growth stress was approximately one-half the value of the experimental measurement. When this film was either in its 'bulk' larger layer thickness $(\lambda=40 \mathrm{~nm})$ or transformed state $(\lambda=2 \mathrm{~nm})$, much closer agreement was found and the film was in a less compressive stress state condition. Even though the non-equal fraction multilayers were shown to be in closer agreement, one can glean from Table 1 that a larger deviation in the calculated and experimental stresses occurred in the more compressive stress states between the $\mathrm{f}_{N b}=0.25$ vs. $\mathrm{f}_{N b}=0.75$ values.

To further understand how the stress evolved in the individual layers, an MD simulation was performed. Fig. 13(a) are the MD simulated growth stresses for a variety of bilayers at $\mathrm{f}_{N b}=$ 0.5. Similar to the computed values using equation (5) and the experimental findings in Fig. 7(b), the MD simulation correctly captured an ever increasing compressive stress with decreasing bilayer with a subsequent stress relaxation. This relaxation also correlated to the simulation predicted change in the fcc-to-bcc $\mathrm{Cu}$ layer. These simulations revealed that the $\mathrm{Nb}$ layers grew more compressive than does $\mathrm{Cu}$ layers, which again was consistent with the experimental trends discussed above. This phase stability and stress value agreement gives confidence that the MD simulation is reasonable in capturing the stress states that evolve within the film. Nonetheless, 
there are some subtle differences particularly with the absolute values between the experimental and simulated growth stresses. These differences are contributed to the simplistic (or idealized) atomic structure used in MD which ignored grain boundary effects, intermixing, and growth environment. Regardless of these differences, the simulated stress values are of the same order of magnitude of the experimental findings and trend in the equivalent manner.

What is particularly interesting in this MD simulation is its ability to capture the stress relaxation between $\lambda=4 \mathrm{~nm}$ and $\lambda=2 \mathrm{~nm}$. By plotting the stress state distribution within the multilayer, Fig. 13(b), we noted that the interface between fcc $\mathrm{Cu} / \mathrm{bcc} \mathrm{Nb}$ at $\lambda=4 \mathrm{~nm}$ was compressive (or blue) in the $\mathrm{Nb}$ layer with the $\mathrm{Cu}$ layer indicating some tensile regions (red atoms) at the interface while the interior of the $\mathrm{Cu}$ film was relatively compressive (or aqua green to blue). When the $\mathrm{Cu}$ layer adopted the bcc phase, Fig. 13(c), a clear onset of tensile stresses (or red) atoms dominated the interface by a near continuous coverage of the interface with $\mathrm{Nb}$. In addition, the interphase is coherent, which is in agreement with the prior HRTEM images. The interior of the bcc $\mathrm{Cu}$ layer now consisted of a distribution of stress states evident by the mosaic of colors. The $\mathrm{Nb}$ layer also revealed some modest changes in stress, apparent by alterations in the atom color states but this was much less prevalent in either its spatial extent in the individual layer or that to the changes the $\mathrm{Cu}$ layer underwent. For completeness, the distribution of the stress states for the 'bulk' fcc $\mathrm{Cu} /$ bcc $\mathrm{Nb}$ multilayer at $\lambda=40 \mathrm{~nm}$ is shown in Fig. 13(d).

Similar to the previous multilayers, the $\mathrm{f}_{N b}=0.25$ also undergoes a stress relaxation with its phase transformation, Fig. 11, but it is much less pronounced and occurred at a layer thickness less than the initial transformation. Though $\mathrm{f}_{N b}=0.25, \lambda=2 \mathrm{~nm}$ exhibited the bcc-to-fcc $\mathrm{Nb}$ transformation, the compressive stress very modestly increased. Only upon further reduction in 
layer thicknesses, where the atom probe data set, Fig. 9(b), confirmed significant intermixing, did the compressive stress relax. The lower compressive stress states for all these films was associated with the lower fraction of $\mathrm{Nb}$ content. The $\mathrm{Nb}$ bcc-to-fcc phase transformation experimentally formed a linear phase boundary, Fig. 10, suggesting negligible strain effects in driving the transformation, which again supports the closer agreement of computed and measured growth stress values tabulated in Table 1 . The lack of a significant change in stress may have also contributed to the existing coherent interface that was present between the two phases in either the bulk or pseudomorphic conditions, Fig. 8.

Based upon the experimental consistent microstructures between the films - grain sizes and columnar morphology across bilayers and volume fractions - coupled with the simplistic predicted growth stress equation as well as targeted MD simulation for $\mathrm{f}_{N b}=0.5$ (which neither deliberately incorporated microstructure), the stress responses in these multilayers appeared to be most significantly affected by the volume fraction of $\mathrm{Nb}$ and the phase of the individual layers.

A final comment is now made concerning the absolute thickness values for these phase transformations and their related residual stress. With changes in deposition rates, growth orientations, or even methods of deposition, it is feasible and even likely the phase transformation thicknesses and stress states will vary. This could be contributed to the change in film's microstructure, including evolving defect structures which develop as the adatoms coalescence and form a continuous film. For example, in the work of Aboulfadl et al. [77] different sputtering rates were used to grow a series of metallic multilayers and a noted change in interfacial intermixing was found as a function of this processing condition. In the computational work by Wang et al. [64], they predicted that different growth rates will modify the threading dislocations that form and change the bcc $\mathrm{Cu}$ stabilization thickness in $\mathrm{Cu} / \mathrm{Nb}$. Such changes, 
wither chemical intermixing, defect formations, and/or strained layers, will then alter the free energies that compose the thermodynamic stability equations described in the introduction. The framework of this thermodynamic construct will still be retained but care in the interpretation of the free energies values that predict the phase stability need to be carefully considered. Thus, the findings reported here are for a specific set of deposition processing parameters. The development of the effects of processing parameters on these thermodynamic energies, such as control of intermixing and defect populations, provides an area of future, fertile research in the area of nanoscale phase stability.

\section{Summary}

In the present study, the phase stability and the corresponding in situ growth stresses of $\mathrm{Cu} / \mathrm{Nb}$ multilayer thin films were investigated. Phases were indentified by XRD and electron diffraction techniques and confirmed both fcc-to-bcc $\mathrm{Cu}$ and bcc-to-fcc $\mathrm{Nb}$ transfromations. Based on a classical thermoldynamic model, a biphase diagram was constructed and linked to the growth stresses. From those links, the following conclusions were drawn:

1. At $\mathrm{f}_{N b}=0.25$ and $\lambda=2 \mathrm{~nm}, \mathrm{fcc} \mathrm{Nb}$ is stabilized in a layered structure. The strain contribution to this stabilization is limited, evident by both a linear phase boundary in the biphase diagram and HRTEM confirmation of a coherent fcc $\mathrm{Cu} / \mathrm{bcc} \mathrm{Nb}$ to coherent fcc $\mathrm{Cu} / \mathrm{fcc} \mathrm{Nb}$ interface. The interfacial energy reduction for this transformation was found to be $\approx-280 \mathrm{mJm}^{-2}$. Additionally our results reveal that the $\mathrm{fcc} \mathrm{Nb}$ stabilization is at a much lower thickness than originally reported and not at an equal volume fraction [29].

2. At $\mathrm{f}_{N b}=0.5$ and 0.75 , the fcc-to-bcc $\mathrm{Cu}$ transformation was noted for $\lambda=2 \mathrm{~nm}$ for both multilayers and the biphase boundary between the bulk and pseudomorphic phase fields 
was curved. This indicated that the transformation is considerably strain dependent and would be consistent with other reports [30, 32, 33].

3. For the $\mathrm{f}_{N b}=0.5 \mathrm{film}$, a reduction of the bilayer thickness to $\lambda=1 \mathrm{~nm}$ vitrified the film indicating yet another transformation step in these multilayers. APT confirmed the loss of the layered interfaces for this glassy film with clusters of $\mathrm{Cu}$ and $\mathrm{Nb}$. For the multilayered materials, where the $\mathrm{Cu}$ was transformed, APT (and HRTEM) confirmed the retention of layered interfaces with APT quantifying that each layer was extensively intermixed.

4. Upon increasing the $\mathrm{Nb}$ content of the multilayer, the overall film stress become more compressive. This was contributed to the highly compressive growth stresses of the $\mathrm{Nb}$ layers; in contrast, Cu's growth stresses were relatively invariant with growth thickness.

5. The phase transformation in all multilayers revealed a relaxation of the compressive growth stresses with the relaxation being the greatest in the $\mathrm{f}_{N b}=0.5$ and 0.75 multilayers. Both a calculated and MD simulation of the stress revealed a similar compressive growth stress behavior with a stress recovery when the fcc-to-bcc $\mathrm{Cu}$ transformed. For the $\mathrm{f}_{N b}=$ 0.5 MD simulation, the $\mathrm{Cu}$ layer revealed dominate tensile stress state at the interface with a distribution of stress states within the film. For the $\mathrm{f}_{N b}=0.25$ multilayer, at the bcc-tofcc $\mathrm{Nb}$ layer, the compressive stress modestly increased but then relaxed with a continuation in reduction of the bilayer spacing, which was contributed to further intermixing between the layer pairs.

6. The use of phase transformations appears to be a means for tunable stress control in nanolaminate multilayered films.

\section{Acknowledgements}


The authors would like to acknowledge the support of the National Science Foundation, DMR-1207220, and the Central Analytical Facility at the University of Alabama in support of this study.

Appendix: A series of figures to supplement the experimental and results discussion presented in the main text.

\section{References:}

[1] L. Berger, Emission of spin waves by a magnetic multilayer traversed by a current, Physical Review B 54(13) (1996) 9353-9358.

[2] P.F. Carcia, A.D. Meinhaldt, A. Suna, Perpendicular magnetic anisotropy in Pd/Co thin film layered structures, Appl. Phys. Lett. 47(2) (1985) 178-180.

[3] M. Tsoi, A.G.M. Jansen, J. Bass, W.C. Chiang, M. Seck, V. Tsoi, P. Wyder, Excitation of a Magnetic Multilayer by an Electric Current, Phys. Rev. Lett. 80(19) (1998) 4281-4284.

[4] A. Bubenzer, B. Dischler, G. Brandt, P. Koidl, rf - plasma deposited amorphous hydrogenated hard carbon thin films: Preparation, properties, and applications, J. Appl. Phys. 54(8) (1983) 4590-4595.

[5] K.K. Shih, D.B. Dove, Ti/Ti - N Hf/Hf - N and W/W - N multilayer films with high mechanical hardness, Appl. Phys. Lett. 61(6) (1992) 654-656.

[6] L. Colace, G. Masini, F. Galluzzi, G. Assanto, G. Capellini, L. Di Gaspare, E. Palange, F. Evangelisti, Metal-semiconductor-metal near-infrared light detector based on epitaxial Ge/Si, Appl. Phys. Lett. 72(24) (1998) 3175-3177.

[7] B. O'Regan, M. Gratzel, A low-cost, high-efficiency solar cell based on dye-sensitized colloidal TiO2 films, Nature 353(6346) (1991) 737-740.

[8] M.J. Demkowicz, D. Bhattacharyya, I. Usov, Y.Q. Wang, M. Nastasi, A. Misra, The effect of excess atomic volume on He bubble formation at fcc-bcc interfaces, Appl. Phys. Lett. 97(16) (2010) 161903.

[9] M.J. Demkowicz, R.G. Hoagland, J.P. Hirth, Interface Structure and Radiation Damage Resistance in Cu-Nb Multilayer Nanocomposites, Phys. Rev. Lett. 100(13) (2008) 136102. [10] J.R. Woodyard, G.A. Landis, Radiation resistance of thin-film solar cells for space photovoltaic power, Solar Cells 31(4) (1991) 297-329.

[11] H.R. Gong, B.X. Liu, Unusual alloying behavior at the equilibrium immiscible $\mathrm{Cu}-\mathrm{Nb}$ interfaces, J. Appl. Phys. 96(5) (2004) 3020-3022.

[12] M.T. Kief, W.F. Egelhoff, Growth and structure of Fe and Co thin films on $\mathrm{Cu}(111)$, $\mathrm{Cu}(100)$, and $\mathrm{Cu}(110)$ : A comprehensive study of metastable film growth, Physical Review B 47(16) (1993) 10785-10814.

[13] R. People, J.C. Bean, Calculation of critical layer thickness versus lattice mismatch for GexSi1-x/Si strained - layer heterostructures, Appl. Phys. Lett. 47(3) (1985) 322-324. [14] A.P. Sutton, R.W. Balluffi, Interfaces in crystalline materials, Clarendon, Oxford, 1995. 
[15] J.H. van der Merwe, G.J. Shiflet, The role of structural ledges at phase boundaries_-III. F.C.C.-B.C.C. interfaces in Kurdjumov-Sachs orientation, Acta Metallurgica et Materialia 42(4) (1994) 1199-1205.

[16] J.S. Bowles, C.M. Wayman, The bain strain, lattice correspondences, and deformations related to martensitic transformations, MT 3(5) (1972) 1113-1121.

[17] R. Hoogeveen, M. Moske, H. Geisler, K. Samwer, Texture and phase transformation of sputter-deposited metastable Ta films and TaCu multilayers, Thin Solid Films 275(1-2) (1996) 203-206.

[18] W.A. Jesser, J.W. Matthews, Evidence for Pseudomorphic Growth of Iron on Copper, Philos. Mag. 15(138) (1967) 1097-\&.

[19] H. Kung, Y.-C. Lu, A.J. Griffin, M. Nastasi, T.E. Mitchell, J.D. Embury, Observation of body centered cubic $\mathrm{Cu}$ in $\mathrm{Cu} / \mathrm{Nb}$ nanolayered composites, Appl. Phys. Lett. 71(15) (1997) 2103-2105.

[20] N. Metoki, W. Donner, H. Zabel, Grazing-incidence x-ray-scattering study of (001)-oriented high-quality epitaxial Co/Cr superlattices, Physical Review B 49(24) (1994) 17351-17359.

[21] B.M. Ocko, I.K. Robinson, M. Weinert, R.J. Randler, D.M. Kolb, Thickness Induced Buckling of bcc Copper Films, Phys. Rev. Lett. 83(4) (1999) 780-783.

[22] Z.Q. Wang, S.H. Lu, Y.S. Li, F. Jona, P.M. Marcus, Epitaxial growth of a metastable modification of copper with body-centered-cubic structure, Physical Review B 35(17) (1987) 9322-9325.

[23] H. Wormeester, E. Hüger, E. Bauer, hcp and bcc Cu and Pd Films, Phys. Rev. Lett. 77(8) (1996) 1540-1543.

[24] J. Wang, A. Misra, An overview of interface-dominated deformation mechanisms in metallic multilayers, Current Opinion in Solid State and Materials Science 15(1) (2011) 20-28. [25] B. Fu, W. An, C.H. Turner, G.B. Thompson, In Situ Thin Film Growth Stresses during Chemical Ordering, Phys. Rev. Lett. 105(9) (2010) 096101.

[26] R. Banerjee, A. Puthucode, S. Bose, P. Ayyub, Nanoscale phase separation in amorphous immiscible copper-niobium alloy thin films, Appl. Phys. Lett. 90(2) (2007) 021904.

[27] P. Boher, F. Giron, P. Houdy, P. Beauvillain, C. Chappert, P. Veillet, Structural and magnetic properties of diode radio - frequency sputtered Cr/Co multilayers, J. Appl. Phys. 70(10) (1991) 5507-5511.

[28] W. Vavra, D. Barlett, S. Elagoz, C. Uher, R. Clarke, Structural transition in epitaxial Co-Cr superlattices, Physical Review B 47(9) (1993) 5500-5503.

[29] J.Y. Zhang, P. Zhang, X. Zhang, R.H. Wang, G. Liu, G.J. Zhang, J. Sun, Mechanical properties of fcc/fcc $\mathrm{Cu} / \mathrm{Nb}$ nanostructured multilayers, Materials Science and Engineering: A 545 (2012) 118-122.

[30] G.B. Thompson, R. Banerjee, S.A. Dregia, H.L. Fraser, Phase stability of bcc Zr in Nb/Zr thin film multilayers, Acta Materialia 51(18) (2003) 5285-5294.

[31] G.B. Thompson, R. Banerjee, H.L. Fraser, Predicting pseudomorphic phases in multilayers: Hexagonal-closed-packed Nb in Nb/Zr, Appl. Phys. Lett. 84(7) (2004) 1082-1084.

[32] R. Bruinsma, A. Zangwill, Structural Transitions in Epitaxial Overlayers, J Phys-Paris 47(12) (1986) 2055-2073.

[33] R. Bruinsma, A. Zangwill, Morphological Transitions in Solid Expitaxial Overlayers, EPL (Europhysics Letters) 4(6) (1987) 729.

[34] R. Banerjee, S.A. Dregia, H.L. Fraser, Stability of f.c.c. titanium in titanium/aluminum multilayers, Acta Materialia 47(15-16) (1999) 4225-4231. 
[35] G.B. Thompson, R. Banerjee, S.A. Dregia, M.K. Miller, H.L. Fraser, A Comparison of Pseudomorphic Bcc Phase Stability in Zr/Nb and Ti/Nb Thin Film Multilayers, J. Mater. Res. 19(03) (2004) 707-715.

[36] S. Dregia, R. Banerjee, H. Fraser, Polymorphic phase stability in thin multilayers, Scripta materialia 39(2) (1998) 217-223.

[37] E. Hahn, E. Kampshoff, N. Wälchli, K. Kern, Strain Driven fcc-bct Phase Transition of Pseudomorphic Cu Films on Pd(100), Phys. Rev. Lett. 74(10) (1995) 1803-1806.

[38] L. Wan, X.-x. Yu, G.B. Thompson, Phase stability and in situ growth stresses in Ti/Nb thin films, Acta Materialia 80 (2014) 490-497.

[39] C. Taylor, D. Barlett, E. Chason, J. Floro, A Laser-Based Thin-Film Growth Monitor, Industrial Physicist 4(1) (1998) 25.

[40] G.G. Stoney, The Tension of Metallic Films Deposited by Electrolysis, Proceedings of the Royal Society of London. Series A, Containing Papers of a Mathematical and Physical Character 82(553) (1909) 172-175.

[41] B.E. Warren, X-ray Diffraction, Courier Corporation1969.

[42] P.J. Felfer, T. Alam, S.P. Ringer, J.M. Cairney, A reproducible method for damage-free site-specific preparation of atom probe tips from interfaces, Microscopy Research and Technique 75(4) (2012) 484-491.

[43] G.B. Thompson, M.K. Miller, H.L. Fraser, Some aspects of atom probe specimen preparation and analysis of thin film materials, Ultramicroscopy 100(1-2) (2004) 25-34.

[44] K. Thompson, D. Lawrence, D.J. Larson, J.D. Olson, T.F. Kelly, B. Gorman, In situ sitespecific specimen preparation for atom probe tomography, Ultramicroscopy 107(2-3) (2007) 131-139.

[45] L.A. Giannuzzi, F.A. Stevie, A review of focused ion beam milling techniques for TEM specimen preparation, Micron 30(3) (1999) 197-204.

[46] J.G. Brons, A.A. Herzing, K.T. Henry, I.M. Anderson, G.B. Thompson, Comparison of atom probe compositional fidelity across thin film interfaces, Thin Solid Films 551 (2014) 61-67. [47] S. Plimpton, Fast parallel algorithms for short-range molecular dynamics, J. Comput. Phys. 117(1) (1995) 1-19.

[48] G.J. Martyna, D.J. Tobias, M.L. Klein, Constant pressure molecular dynamics algorithms, The Journal of Chemical Physics 101(5) (1994) 4177-4189.

[49] M. Parrinello, A. Rahman, Polymorphic transitions in single crystals: A new molecular dynamics method, J. Appl. Phys. 52(12) (1981) 7182-7190.

[50] M.E. Tuckerman, J. Alejandre, R. López-Rendón, A.L. Jochim, G.J. Martyna, A Liouvilleoperator derived measure-preserving integrator for molecular dynamics simulations in the isothermal-isobaric ensemble, Journal of Physics A: Mathematical and General 39(19) (2006) 5629.

[51] W. Shinoda, M. Shiga, M. Mikami, Rapid estimation of elastic constants by molecular dynamics simulation under constant stress, Physical Review B 69(13) (2004) 134103. [52] A. Stukowski, Visualization and analysis of atomistic simulation data with OVITO-the Open Visualization Tool, Modelling and Simulation in Materials Science and Engineering 18(1) (2009) 015012.

[53] J.D. Honeycutt, H.C. Andersen, Molecular dynamics study of melting and freezing of small Lennard-Jones clusters, J. phys. Chem 91(19) (1987) 4950-4963.

[54] E.E. Fullerton, I.K. Schuller, H. Vanderstraeten, Y. Bruynseraede, Structural refinement of superlattices from x-ray diffraction, Physical Review B 45(16) (1992) 9292. 
[55] I.K. Schuller, New class of layered materials, Phys. Rev. Lett. 44(24) (1980) 1597.

[56] G.V. Khaldeev, Physical and Corrosion-electrochemical Properties of the NiobiumHydrogen System, Russian chemical reviews 56(7) (1987) 605.

[57] G. Thompson, R. Banerjee, H. Fraser, Specimen Dependent Milling Directions for Correct Phase Identification in Multilayered Thin Films, Microscopy and Microanalysis 9(S02) (2003) 370-371.

[58] J. Goedkoop, A. Andresen, The crystal structure of copper hydride, Acta Crystallogr. 8(2) (1955) 118-119.

[59] S. Labat, F. Bocquet, B. Gilles, O. Thomas, Stresses and interfacial structure in Au-Ni and $\mathrm{Ag}-\mathrm{Cu}$ metallic multilayers, Scripta materialia 50(6) (2004) 717-721.

[60] F. Spaepen, Interfaces and stresses in thin films, Acta Materialia 48(1) (2000) 31-42.

[61] A.L. Shull, F. Spaepen, Measurements of stress during vapor deposition of copper and silver thin films and multilayers, J. Appl. Phys. 80(11) (1996) 6243-6256.

[62] L.Z. Mezey, J. Giber, The Surface Free Energies of Solid Chemical Elements: Calculation from Internal Free Enthalpies of Atomization, Jpn. J. Appl. Phys. 21(11R) (1982) 1569.

[63] N. Li, J. Wang, A. Misra, J.Y. Huang, Direct observations of confined layer slip in cu/nb multilayers, Microscopy and microanalysis 18(05) (2012) 1155-1162.

[64] J. Wang, R.G. Hoagland, A. Misra, Phase transition and dislocation nucleation in $\mathrm{Cu}-\mathrm{Nb}$ layered composites during physical vapor deposition, J. Mater. Res. 23(04) (2008) 1009-1014.

[65] M.J. Demkowicz, R.G. Hoagland, Structure of Kurdjumov-Sachs interfaces in simulations of a copper-niobium bilayer, J. Nucl. Mater. 372(1) (2008) 45-52.

[66] A. Genç, Phase Stability in Metallic Multilayers, Materials Science and Engineering, The Ohio State University, 2008, p. 198.

[67] K. Coffey, K. Barmak, A new model for grain boundary diffusion and nucleation in thin film reactions, Acta metallurgica et materialia 42(8) (1994) 2905-2911.

[68] S. Bose, A. Puthucode, R. Banerjee, P. Ayyub, The influence of nanoscale phase separation and devitrification on the electrical transport properties of amorphous $\mathrm{Cu}-\mathrm{Nb}$ alloy thin films, Journal of Physics: Condensed Matter 21(28) (2009) 285305.

[69] A. Puthucode, A. Devaraj, S. Nag, S. Bose, P. Ayyub, M. Kaufman, R. Banerjee, Devitrification of nanoscale phase-separated amorphous thin films in the immiscible copperniobium system, Philos. Mag. 94(15) (2014) 1622-1641.

[70] A. Puthucode, M.J. Kaufman, R. Banerjee, Early Stages of Crystallization in PhaseSeparated Amorphous Copper-Niobium Alloy Thin Films, MMTA 39(7) (2008) 1578-1584. [71] B. Sundman, B. Jansson, J.-O. Andersson, The Thermo-Calc databank system, Calphad 9(2) (1985) 153-190.

[72] J.M. Slaughter, A. Shapiro, P.A. Kearney, C.M. Falco, Growth of molybdenum on silicon: Structure and interface formation, Physical Review B 44(8) (1991) 3854-3863.

[73] R. Banerjee, S. Bose, A. Genc, P. Ayyub, The microstructure and electrical transport properties of immiscible copper-niobium alloy thin films, J. Appl. Phys. 103(3) (2008) 033511. [74] A. Fartash, E.E. Fullerton, I.K. Schuller, S.E. Bobbin, J.W. Wagner, R.C. Cammarata, S. Kumar, M. Grimsditch, Evidence for the supermodulus effect and enhanced hardness in metallic superlattices, Physical Review B 44(24) (1991) 13760-13763.

[75] V. Ramaswamy, W. Nix, B. Clemens, Coherency and surface stress effects in metal multilayers, Scripta materialia 50(6) (2004) 711-715.

[76] A. Misra, J.P. Hirth, R.G. Hoagland, Length-scale-dependent deformation mechanisms in incoherent metallic multilayered composites, Acta Materialia 53(18) (2005) 4817-4824. 
[77] H. Aboulfadl, I. Gallino, R. Busch, F. Mücklich, Atomic scale analysis of phase formation and diffusion kinetics in Ag/Al multilayer thin films, J. Appl. Phys. 120(19) (2016) 195306. 


\section{TABLE CAPTIONS}

Table 1: Tabulation of calculated and measured growth stress per unit length values for the various $\mathrm{Nb}$ fraction and bilayer spacing multilayers.

\section{FIGURE CAPTIONS}

Fig. 1: A proposed biphase stability diagram based on a previously report [19].

Fig. 2: $\mathrm{X}$-ray diffraction spectra of $\mathrm{Cu} / \mathrm{Nb}$ multilayered film with different bilayer thickness $(\lambda)$ at (a) $\mathrm{f}_{N b}=0.25$ (b) $\mathrm{f}_{N b}=0.5$ and (c) $\mathrm{f}_{N b}=0.75$. The dash line represents the standard bulk powder location for each phase for the labeled $\{\mathrm{hkl}\}$ reflection and the red arrow represents satellite peaks.

Fig. 3: Electron diffraction pattern taken under plan-view orientation of representative multilayered thin film with $\mathrm{Nb}$ volume fraction $\mathrm{f}_{N b}=0.25$. (a) Diffraction pattern of $\lambda=40 \mathrm{~nm}$ foil. (b) Diffraction pattern of $\lambda=4 \mathrm{~nm}$ foil. (c) Diffraction pattern of $\lambda=2 \mathrm{~nm}$ foil. (d) Diffraction pattern of $\lambda=1.2 \mathrm{~nm}$ foil.

Fig. 4: Electron diffraction pattern taken under plan-view orientation of representative multilayered thin film with $\mathrm{Nb}$ volume fraction $\mathrm{f}_{N b}=0.5$. (a) Diffraction pattern of $\lambda=40 \mathrm{~nm}$ foil. (b) Diffraction pattern of $\lambda=4 \mathrm{~nm}$ foil. (c) Diffraction pattern of $\lambda=2 \mathrm{~nm}$ foil. (d) Diffraction pattern of $\lambda=1 \mathrm{~nm}$ foil.

Fig. 5: Electron diffraction pattern taken under plan-view orientation of representative multilayered thin film with $\mathrm{Nb}$ volume fraction $\mathrm{f}_{N b}=0.75$. (a) Diffraction pattern of $\lambda=40 \mathrm{~nm}$ foil. (b) Diffraction pattern of $\lambda=4 \mathrm{~nm}$ foil. (c) Diffraction pattern of $\lambda=2 \mathrm{~nm}$ foil. (d) Diffraction pattern of $\lambda=1.2 \mathrm{~nm}$ foil.

Fig. 6: Cross sectional images of (a) Bright field TEM $\lambda=40 \mathrm{~nm} / \mathrm{f}_{N b}=0.25$ multilayer (inset image is a STEM-High Angle Annular Dark Field (HAADF) image) (b) STEM-HAADF $\lambda=10$ $\mathrm{nm} / \mathrm{f}_{N b}=0.5$ (c) Bright field TEM of $\lambda=2 \mathrm{~nm} / \mathrm{f}_{N b}=0.5$ with the inset image confirming the layered morphology.

Fig. 7: (a) Stress evolution of the stress-thickness product verses thickness for $\mathrm{Nb}$ volume fraction $\mathrm{f}_{N b}=0.25$ multilayers listed by their bilayer thickness $(\lambda)$. (b) Stress evolution of the stress-thickness product verses thickness for $\mathrm{Nb}$ volume fraction $\mathrm{f}_{N b}=0.5$ multilayers listed by their bilayer thickness $(\lambda)$. The inset image shows the growth stress oscillations as each layer grows. (c) Stress evolution of the stress-thickness product verses thickness for $\mathrm{Nb}$ volume fraction $\mathrm{f}_{N b}=0.75$ multilayers listed by their bilayer thickness $(\lambda)$. (d) Magnified image of $\mathrm{f}_{N b}=$ $0.25, \lambda=4 \mathrm{~nm}$ 'bulk' phase multilayers. (e) Magnified image of $\mathrm{f}_{N b}=0.5, \lambda=4 \mathrm{~nm}$ 'bulk' phase multilayers. (f) Magnified image of $\mathrm{f}_{N b}=0.75, \lambda=4 \mathrm{~nm}$ 'bulk' phase multilayers. (g) Magnified 
image of $\mathrm{f}_{N b}=0.25, \lambda=2 \mathrm{~nm}$ phase transformed multilayers. (h) Magnified image of $\mathrm{f}_{N b}=0.5, \lambda$ $=2 \mathrm{~nm}$ phase transformed multilayers. (i) Magnified image of $\mathrm{f}_{N b}=0.75, \lambda=2 \mathrm{~nm}$ phase transformed multilayers. Color available online.

Fig. 8: (a) HR-TEM cross-section image of $\lambda=40 \mathrm{~nm} / \mathrm{f}_{N b}=0.25$ multilayers at interface with the present of misfit dislocations and semi-coherent interface. Thin dash line indicates the extra halfplane (b) HR-TEM cross-section image of $\lambda=4 \mathrm{~nm} / \mathrm{f}_{N b}=0.25$ multilayers at interface with the present of distorted lattice at coherent interface (indicated by yellow, red and dark blue lines). (c) HR-TEM cross-section image of $\lambda=2 \mathrm{~nm} / \mathrm{f}_{N b}=0.25$ multilayers at interface with the present of both no-distortion (indicated by green lines) and distortion coherent interfaces (indicated by red lines). (d) HR-TEM cross-section image of $\lambda=1.2 \mathrm{~nm} / \mathrm{f}_{N b}=0.25$ multilayers at interface with the present of no-distortion coherent interfaces (indicated by green lines). Color available online.

Fig. 9: (a) 1D concentration profile across the interfaces of the $\lambda=2 \mathrm{~nm} / \mathrm{f}_{N b}=0.5$ multilayer with atom ion map inserted. (b) Spatial distribution map of $\mathrm{Cu}$ atoms of $\lambda=1.2 \mathrm{~nm} / \mathrm{f}_{N b}=0.25$ multilayer with atom ion map inserted. (c) Spatial distribution map of $\mathrm{Cu}$ atoms of $\lambda=1.2$ $\mathrm{nm} / \mathrm{f}_{N b}=0.75$ multilayer with atom ion map inserted. (d) Atom ion map of region of interest (ROI) and related $\mathrm{Cu}$ and $\mathrm{Nb}$ cluster maps of $\lambda=1 \mathrm{~nm} / \mathrm{f}_{N b}=0.5$ multilayer. Color available online.

Fig. 10: Phase stability diagram. The dash lines represent the original predictions for phase space whereas the solid lines now represent the new experimental boundaries created by this report. Filled rectangular represents multilayers with fcc $\mathrm{Cu} / \mathrm{bcc} \mathrm{Nb}$ phases. Filled circle represents multilayers with fcc $\mathrm{Cu} / \mathrm{fcc} \mathrm{Nb}$ phases. Up triangle represents multilayers with bcc $\mathrm{Cu} / \mathrm{bcc} \mathrm{Nb}$ phases. Down triangle represents multilayers with amorphous phase. Solid and dashed line represents phase boundary of different phases.

Fig. 11: Growth stress versus bilayer thickness curves for (a) $\mathrm{f}_{N b}=0.25$, (b) $\mathrm{f}_{N b}=0.5$ and (c) $\mathrm{f}_{N b}$ $=0.75 \mathrm{Cu} / \mathrm{Nb}$ multilayers. Solid arrow represents the formation fcc $\mathrm{Nb}$ phase. Hollow arrows represent the formation of bcc $\mathrm{Cu}$ phase. Slashed arrow represents the formation of $\mathrm{Cu} / \mathrm{Nb}$ amorphous phase.

Fig. 12: HR-TEM cross-section image of $\lambda=2 \mathrm{~nm} / \mathrm{f}_{N b}=0.5$ multilayers at interface with the present of no-distortion coherent interfaces (indicated by green lines). Color available online.

Fig. 13: MD simulation of $\mathrm{f}_{N b}=0.5$ for various bilayer spacings (a) Overall growth stress (b) atomic stress state distrubtion in fcc $\mathrm{Cu} / \mathrm{bcc} \mathrm{Nb}$ at $\lambda=4 \mathrm{~nm}$ (c) atomic stress state distrubtion in bcc Cu/bcc Nb at $\lambda=2 \mathrm{~nm}$ (d) atomic stress state distrubtion in fcc $\mathrm{Cu} / \mathrm{bcc} \mathrm{Nb}$ at $\lambda=40 \mathrm{~nm}$.

Fig. A1: Representative plan-view precession electron diffraction (PED) scans and inverse pole figures of labeled bilayers with corresponding grain size histograms under each scanned region.

Fig. A2: A representative atom of $1 \mathrm{~nm} \mathrm{Cu} / 1 \mathrm{~nm} \mathrm{Nb}$ with its accompanying time-of-flight mass spectrum. Note that no argon or oxygen was detected in the film. The presence of hydrogen is a known vacuum containment gas and believed not to have stabilized the phases evident by the diffraction results and measured lattice parameter discussed in the paper. 


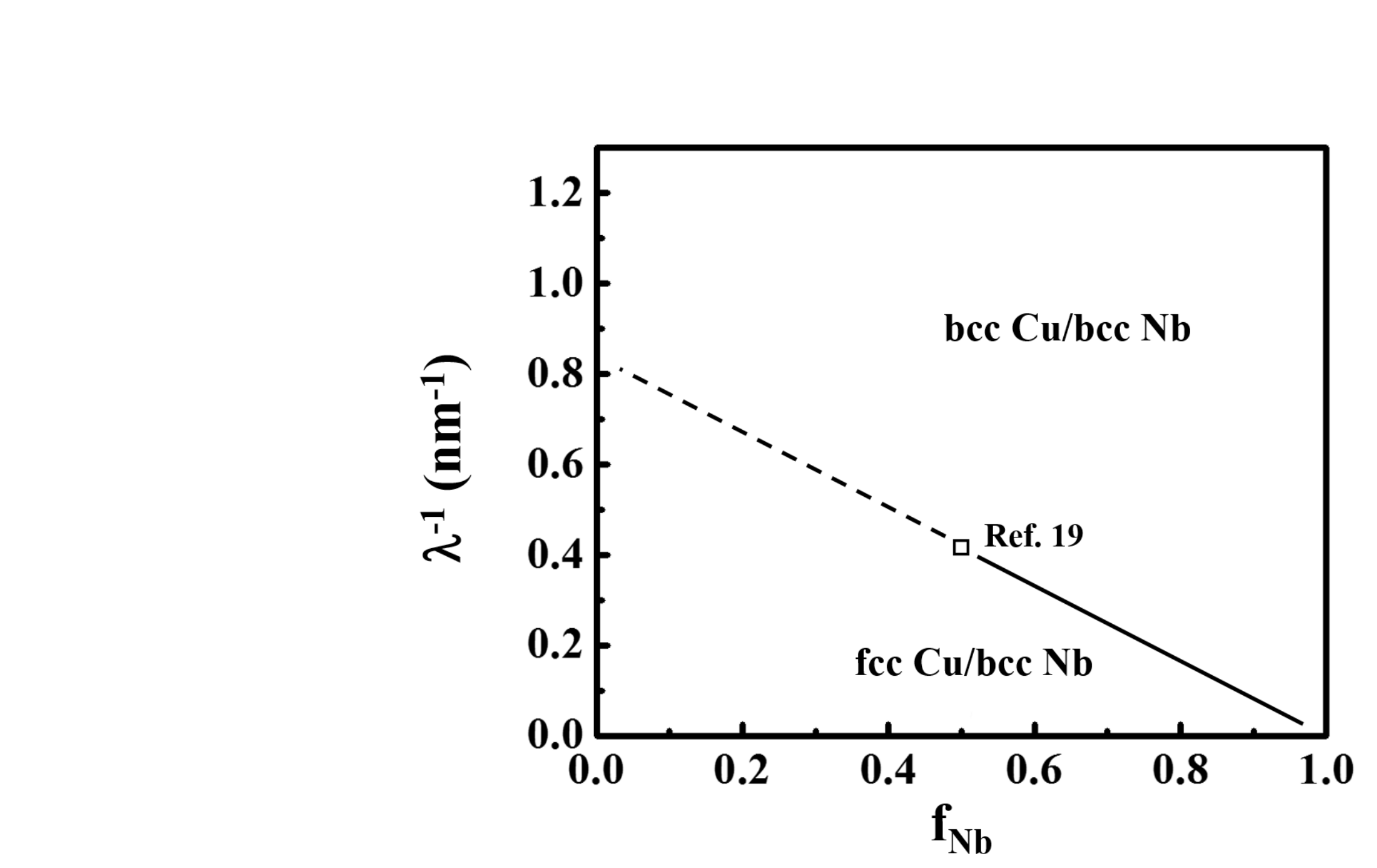

Fig. 1: A proposed biphase stability diagram based on a previously report [19]. .

ort [19].

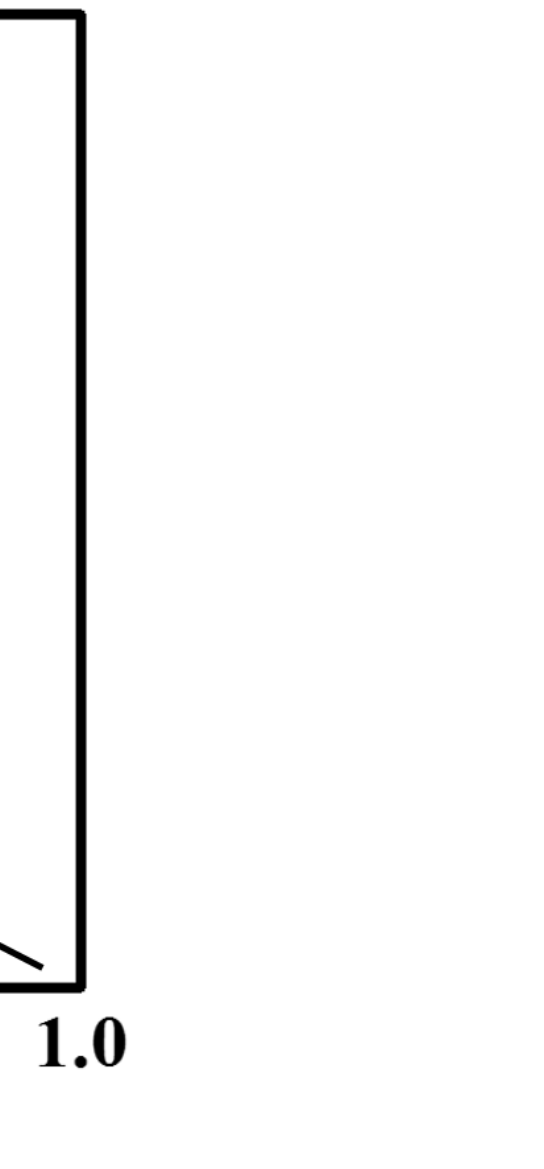

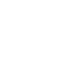
$\sqrt{2}$ 

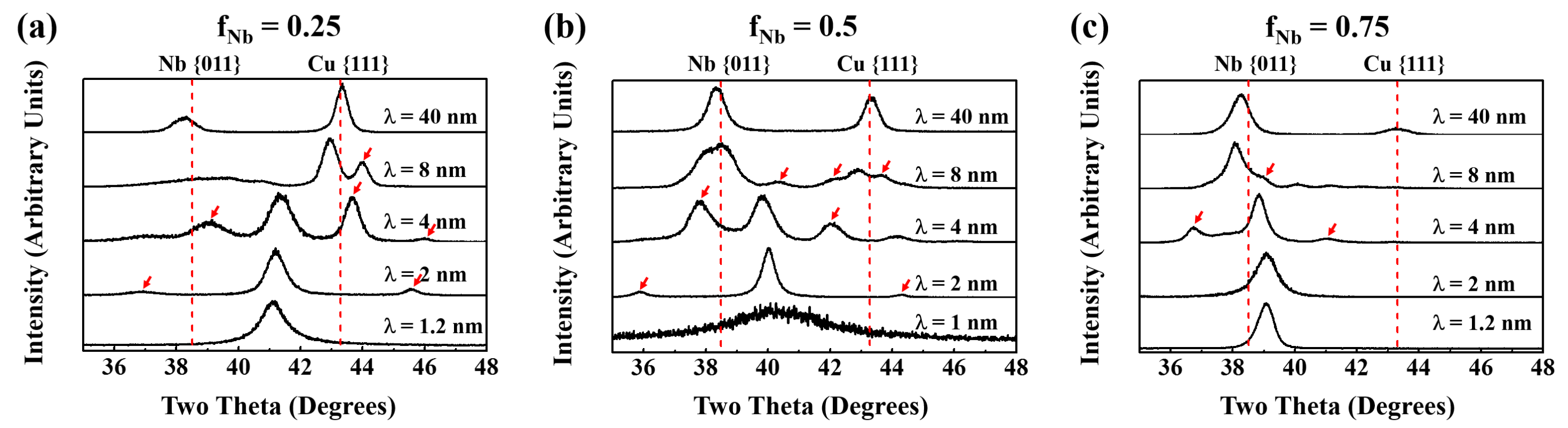

Fig. 2: X-ray diffraction spectra of $\mathrm{Cu} / \mathrm{Nb}$ multilayered film with different bilayer thickness $(\lambda)$ at $(\mathrm{a}) \mathrm{f}_{N b}=0.25$ (b) $\mathrm{f}_{N b}=$ 0.5 and (c) $\mathrm{f}_{N b}=0.75$. The dash line represents the standard bulk powder location for each phase for the labeled $\{\mathrm{hkl}\}$ reflection and the red arrow represents satellite peaks. 

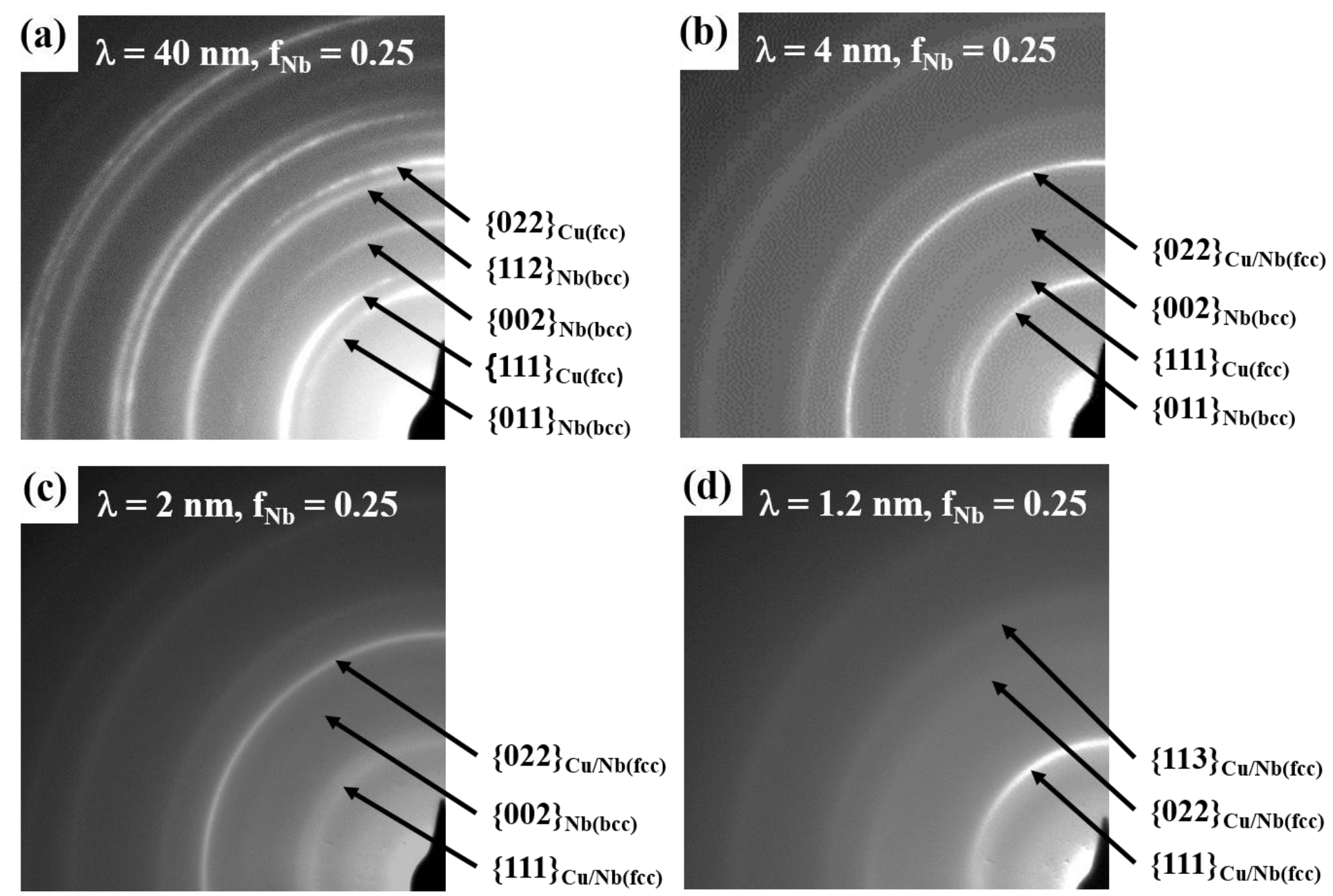

Fig. 3: Electron diffraction pattern taken under plan-view orientation of representative multilayered thin film with $\mathrm{Nb}$ volume fraction $\mathrm{f}_{N b}=0.25$. (a) Diffraction pattern of $\lambda=40 \mathrm{~nm}$ foil. (b) Diffraction pattern of $\lambda=4 \mathrm{~nm}$ foil. (c) Diffraction pattern of $\lambda=2 \mathrm{~nm}$ foil. (d) Diffraction pattern of $\lambda=1.2 \mathrm{~nm}$ foil. 

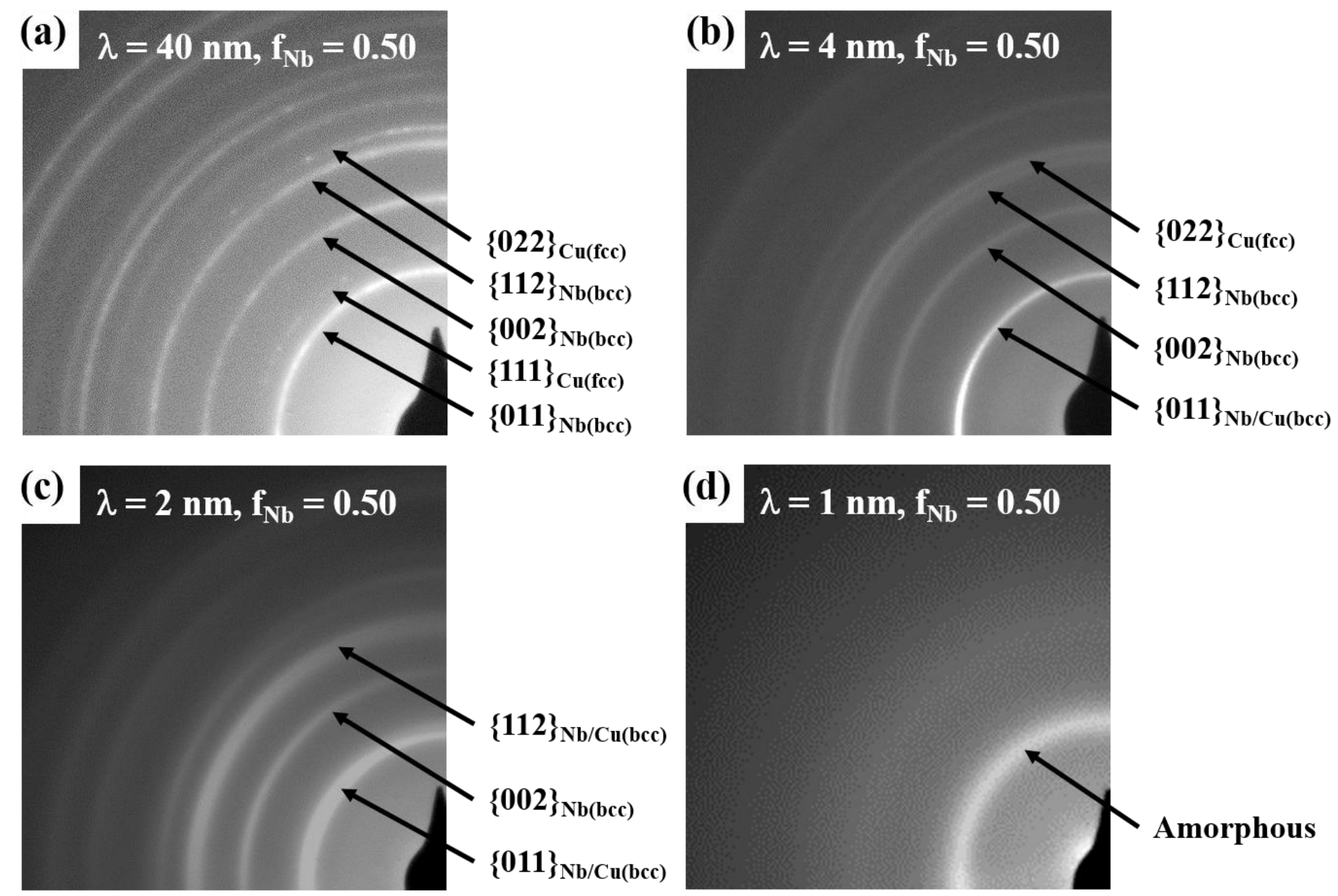

(d) $\lambda=1 \mathrm{~nm}, \mathrm{f}_{\mathrm{Nb}}=0.50$

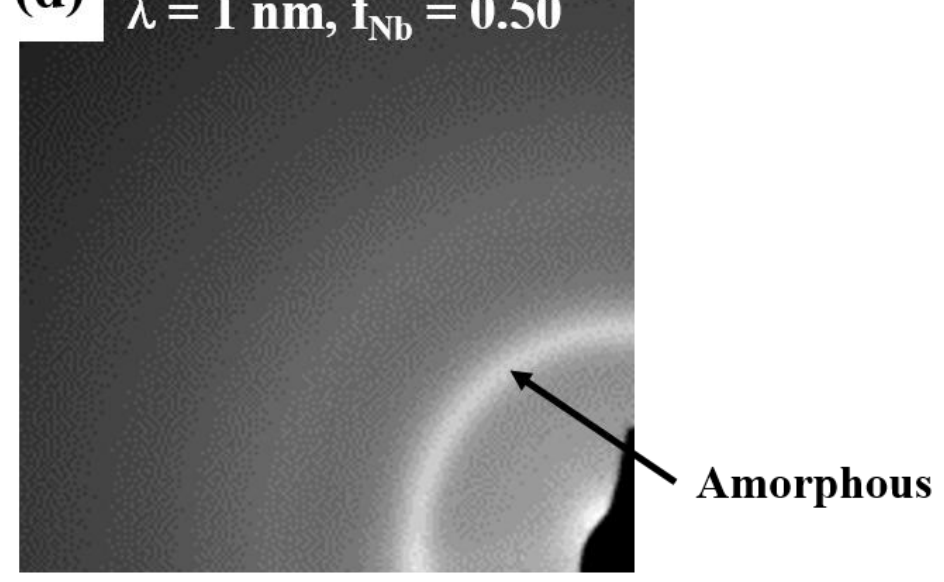

Fig. 4: Electron diffraction pattern taken under plan-view orientation of representative multilayered thin film with $\mathrm{Nb}$ volume fraction $\mathrm{f}_{N b}=0.5$. (a) Diffraction pattern of $\lambda=40 \mathrm{~nm}$ foil. (b) Diffraction pattern of $\lambda=4 \mathrm{~nm}$ foil. (c) Diffraction pattern of $\lambda=2 \mathrm{~nm}$ foil. (d) Diffraction pattern of $\lambda=1 \mathrm{~nm}$ foil. 


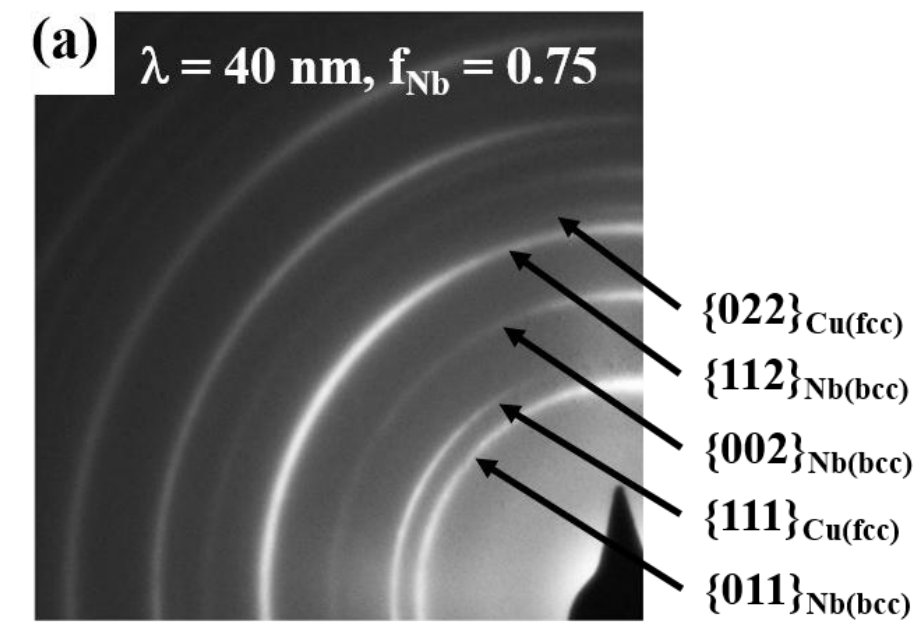

(b) $\lambda=4 \mathrm{~nm}, \mathrm{f}_{\mathrm{Nb}}=0.75$
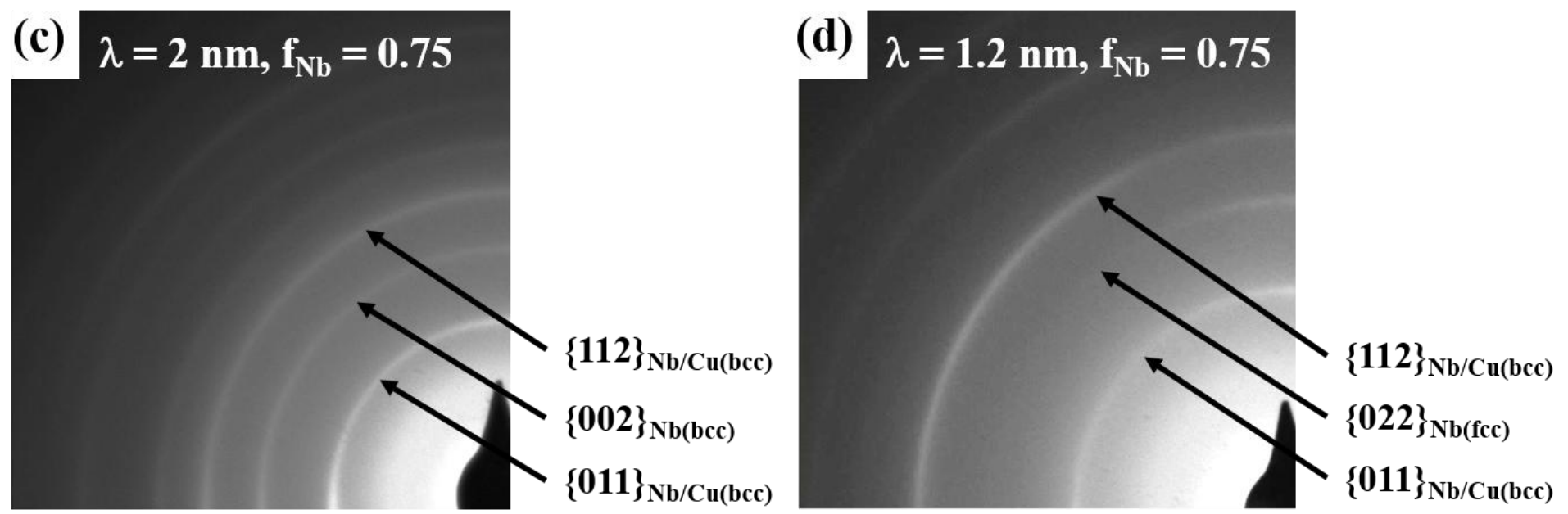

Fig. 5: Electron diffraction pattern taken under plan-view orientation of representative multilayered thin film with $\mathrm{Nb}$ volume fraction $\mathrm{f}_{N b}=0.75$. (a) Diffraction pattern of $\lambda=40 \mathrm{~nm}$ foil. (b) Diffraction pattern of $\lambda=4 \mathrm{~nm}$ foil. (c) Diffraction pattern of $\lambda=2 \mathrm{~nm}$ foil. (d) Diffraction pattern of $\lambda=1.2 \mathrm{~nm}$ foil. 


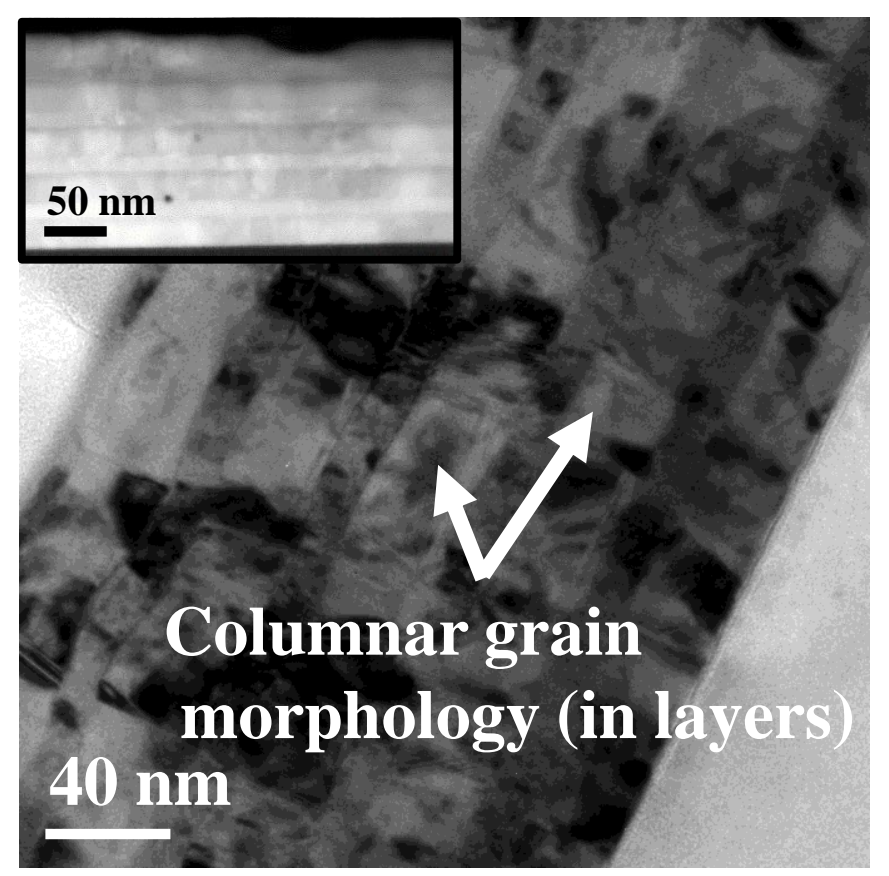

$\lambda=40 \mathrm{~nm}, \mathrm{f}_{\mathrm{Nb}}=0.25$

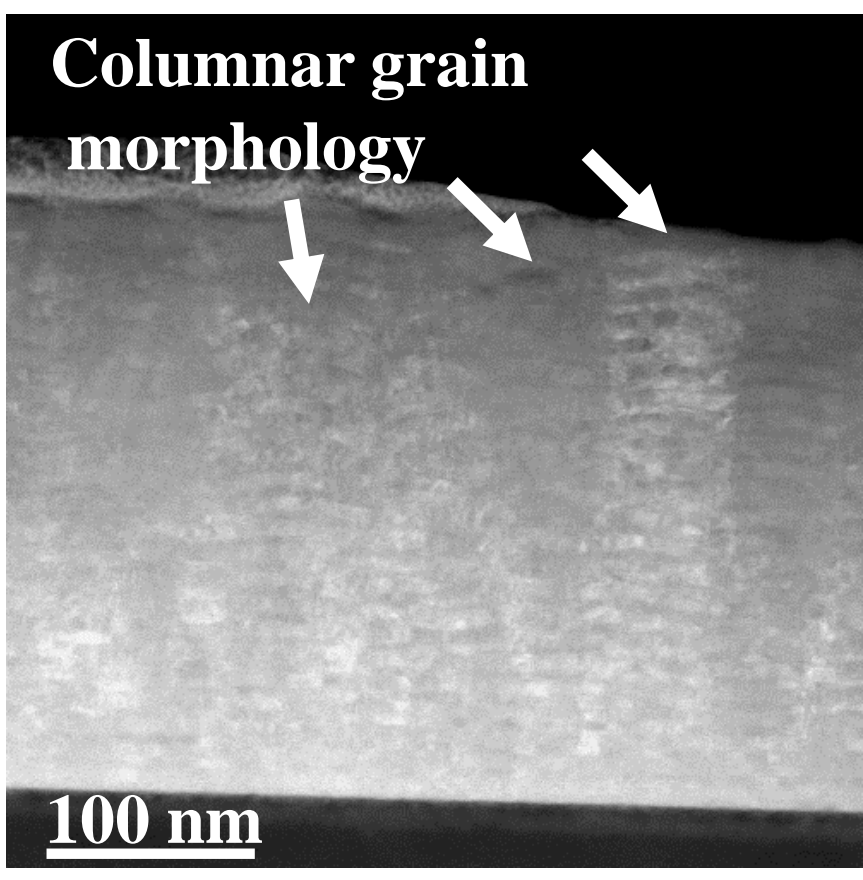

$\lambda=10 \mathrm{~nm}, \mathbf{f}_{\mathrm{Nb}}=0.5$

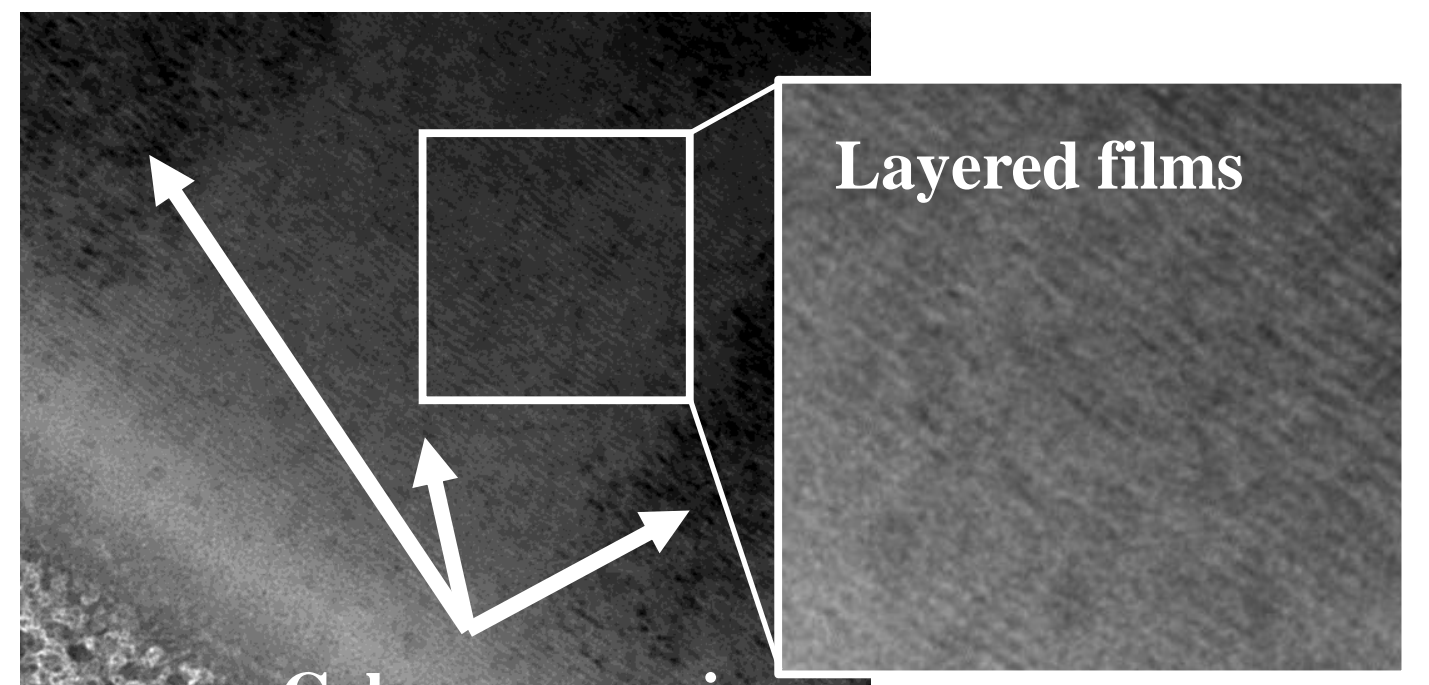

Columnar grain

\section{0 nim morphology}

$$
\lambda=2 \mathrm{~nm}, \mathrm{f}_{\mathrm{Nb}}=0.5
$$

Fig. 6: Cross sectional images of (a) Bright field TEM $\lambda=40 \mathrm{~nm} / \mathrm{f}_{N b}=0.25$ multilayer (inset image is a STEM-High Angle Annular Dark Field (HAADF) image) (b) STEM-HAADF $\lambda=10 \mathrm{~nm} / \mathrm{f}_{N b}=0.5$ (c) Bright field TEM of $\lambda=2 \mathrm{~nm} / \mathrm{f}_{N b}=0.5$ with the inset image confirming the layered morphology. 
(a)

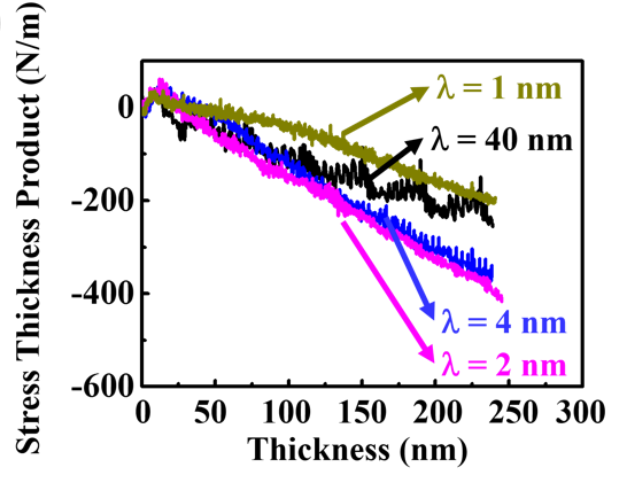

(b) 㐭

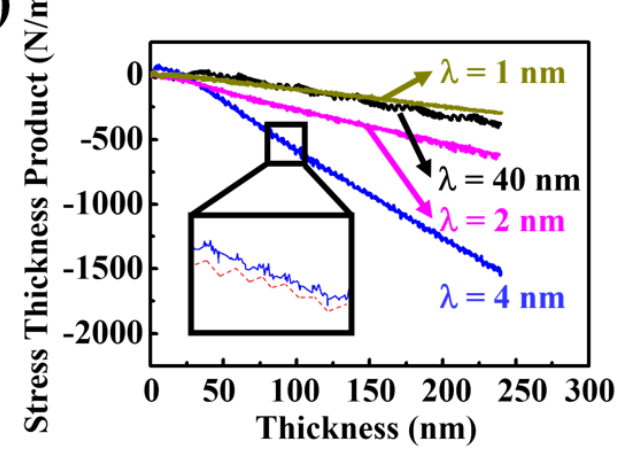

(c) $\widehat{\Xi}$

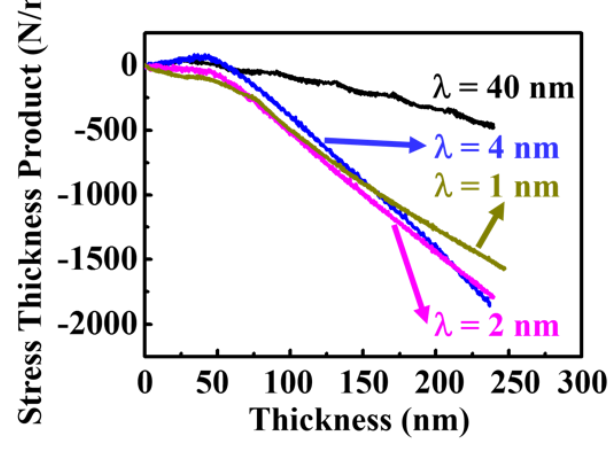

(d) $\widehat{\Xi}$

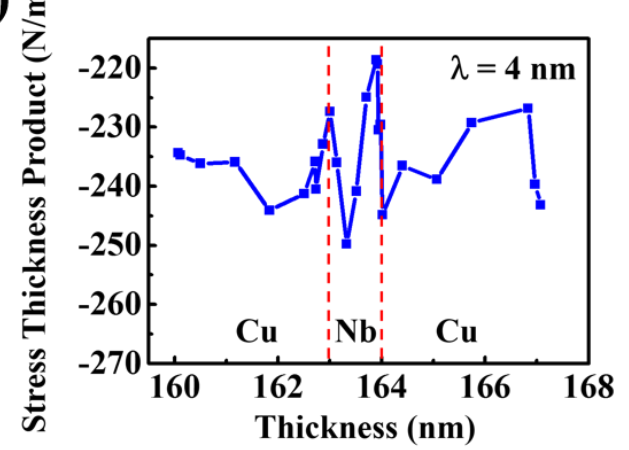

(e) $\widehat{\Xi}$

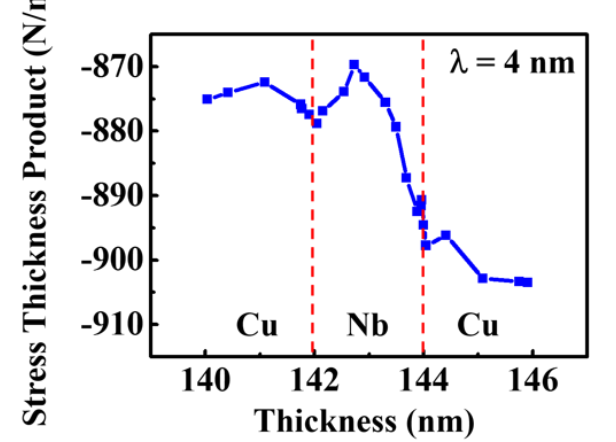

(f)

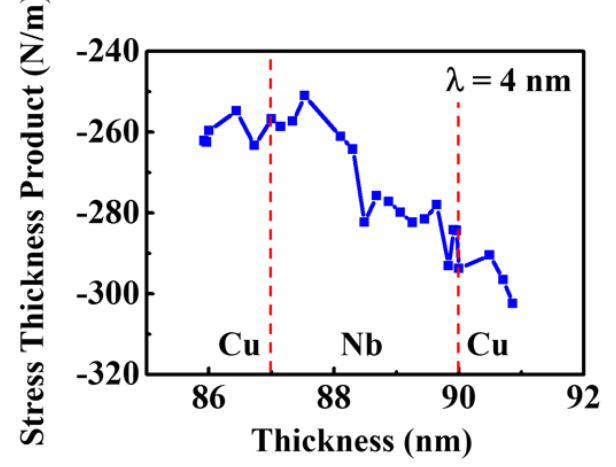

(g)

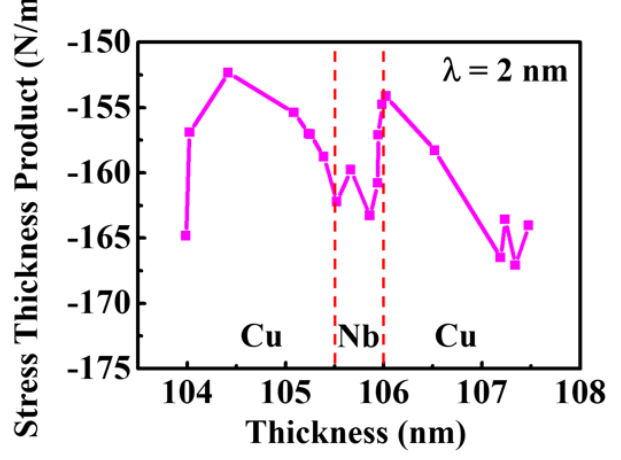

(h) 要

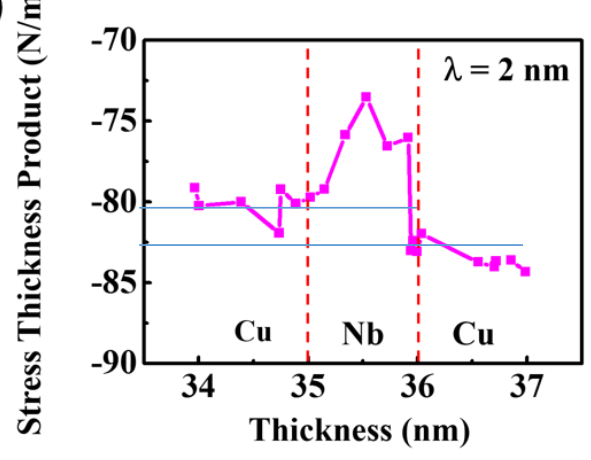

(i) $\bar{\Xi}$

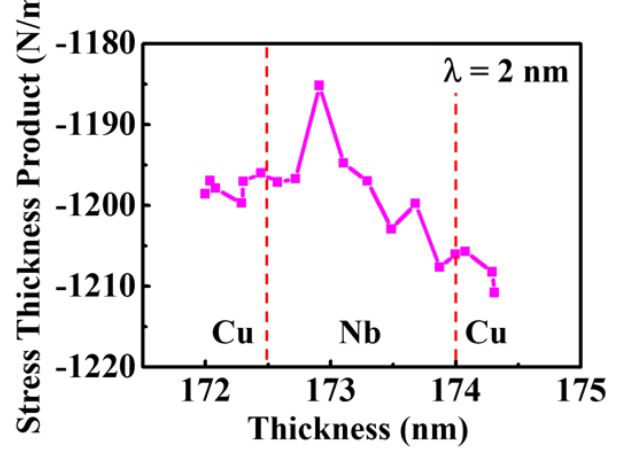

Fig. 7: (a) Stress evolution of the stress-thickness product verses thickness for Nb volume fraction $\mathrm{f}_{N b}=0.25$ multilayers listed by their bilayer thickness $(\lambda$ ). (b) Stress evolution of the stress-thickness product verses thickness for $\mathrm{Nb}$ volume fraction $\mathrm{f}_{N b}=0.5$ multilayers listed by their bilayer thickness $(\lambda)$. The inset image shows the growth stress oscillations as each layer grows. (c) Stress evolution of the stress-thickness product verses thickness for $\mathrm{Nb}$ volume fraction $\mathrm{f}_{N b}=0.75$ multilayers listed by their bilayer thickness $(\lambda)$. (d) Magnified image of $\mathrm{f}_{N b}=0.25, \lambda=4 \mathrm{~nm}$ 'bulk' phase multilayers. (e) Magnified image of $\mathrm{f}_{N b}=0.5, \lambda=4$ nm 'bulk' phase multilayers. (f) Magnified image of $\mathrm{f}_{N b}=0.75, \lambda=4 \mathrm{~nm}$ 'bulk' phase multilayers. (g) Magnified image of $\mathrm{f}_{N b}=0.25, \lambda=2$ nm phase transformed multilayers. (h) Magnified image of $\mathrm{f}_{N b}=0.5, \lambda=2 \mathrm{~nm}$ phase transformed multilayers. (i) Magnified image of $\mathrm{f}_{N b}=0.75, \lambda=2 \mathrm{~nm}$ phase transformed multilayers. Color available online. 
(a)

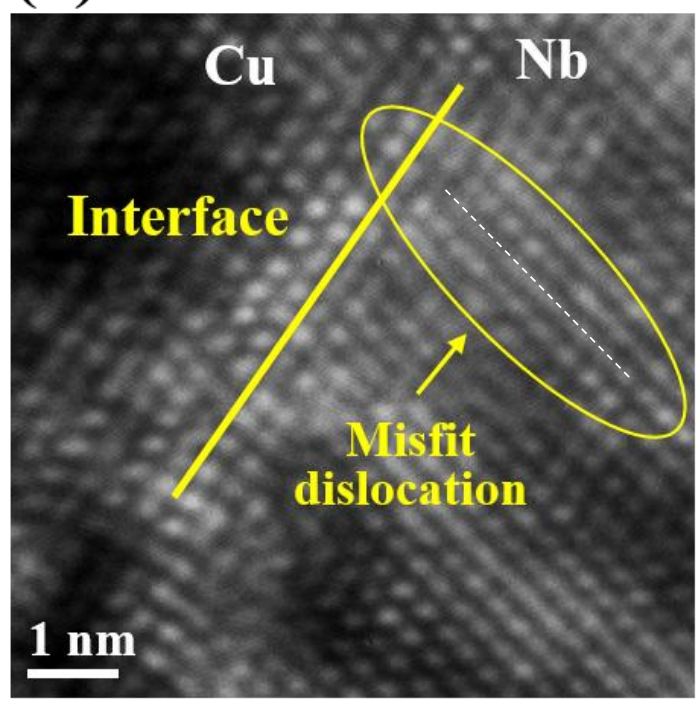

$$
\lambda=40 \mathrm{~nm}, \mathbf{f}_{\mathrm{Nb}}=0.25
$$

(b)

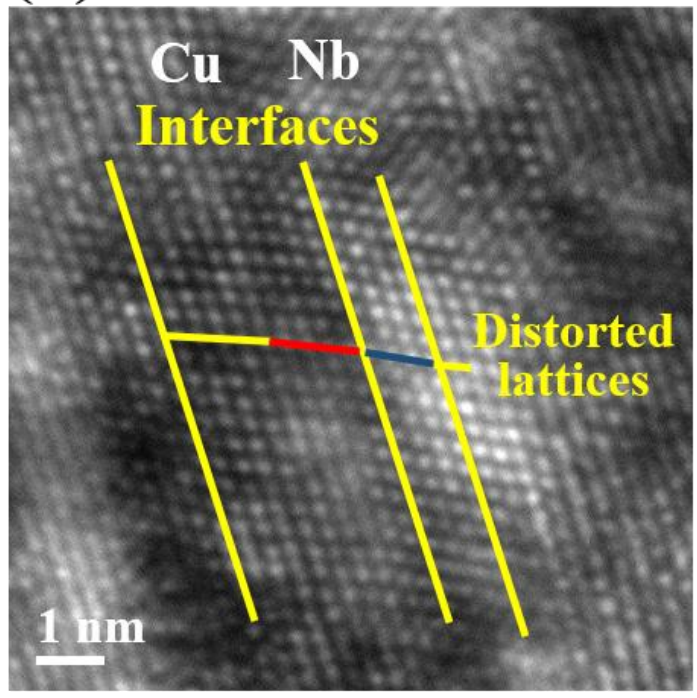

$$
\lambda=4 \mathrm{~nm}, \mathrm{f}_{\mathrm{Nb}}=0.25
$$

(c)

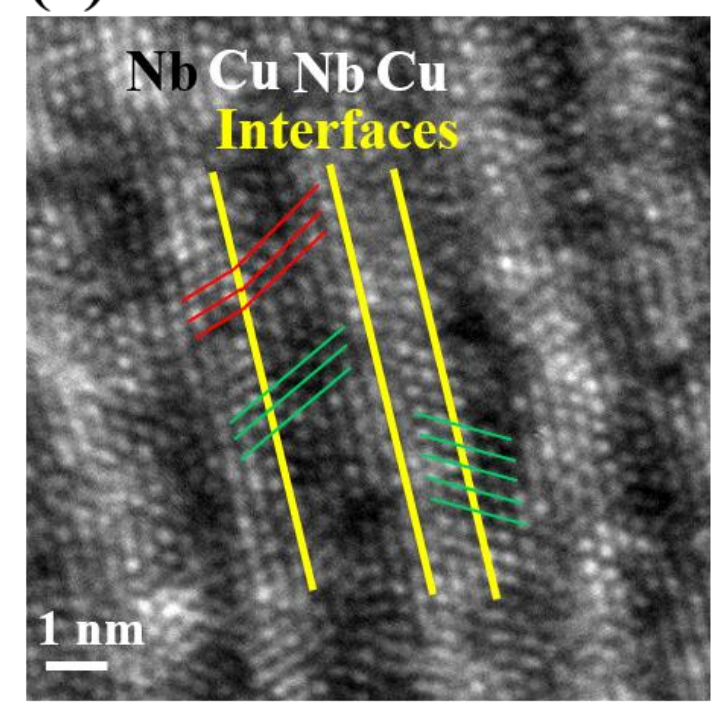

$$
\lambda=2 \mathrm{~nm}, \mathrm{f}_{\mathrm{Nb}}=0.25
$$

(d)

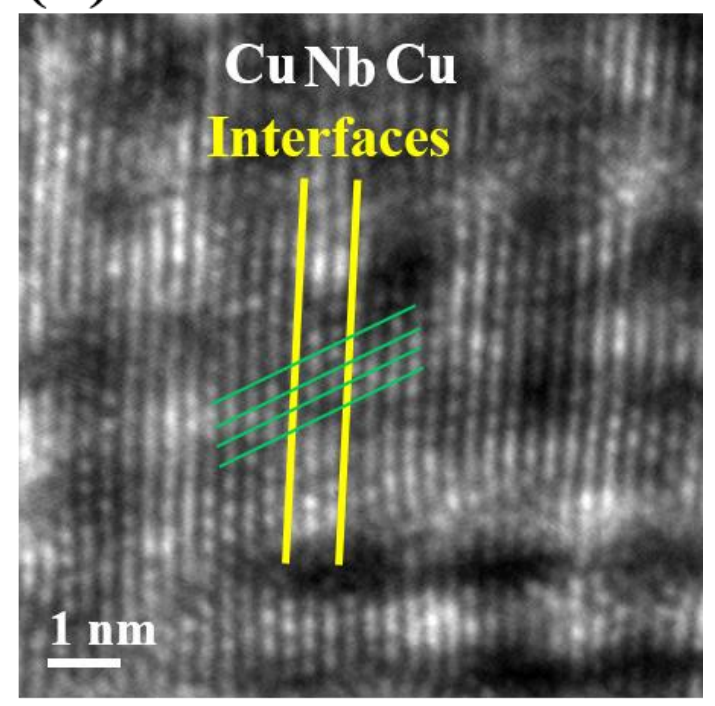

$\lambda=1.2 \mathrm{~nm}, \mathbf{f}_{\mathrm{Nb}}=0.25$

Fig. 8: (a) HR-TEM cross-section image of $\lambda=40 \mathrm{~nm} / \mathrm{f}_{N b}=0.25$ multilayers at interface with the present of misfit dislocations and semi-coherent interface. Thin dash line indicates the extra half-plane (b) HR-TEM cross-section image of $\lambda$ $=4 \mathrm{~nm} / \mathrm{f}_{N b}=0.25$ multilayers at interface with the present of distorted lattice at coherent interface (indicated by yellow, red and dark blue lines). (c) HR-TEM cross-section image of $\lambda=2 \mathrm{~nm} / \mathrm{f}_{N b}=0.25$ multilayers at interface with the present of both no-distortion (indicated by green lines) and distortion coherent interfaces (indicated by red lines). (d) HR-TEM crosssection image of $\lambda=1.2 \mathrm{~nm} / \mathrm{f}_{N b}=0.25$ multilayers at interface with the present of no-distortion coherent interfaces (indicated by green lines). Color available online. 
(a)

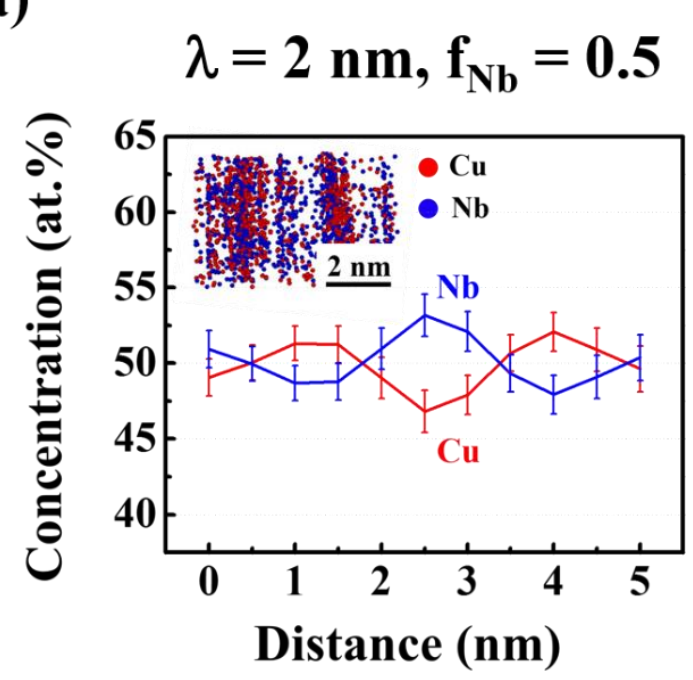

(c)

$$
\lambda=1.2 \mathrm{~nm}, \mathrm{f}_{\mathrm{Nb}}=0.75
$$

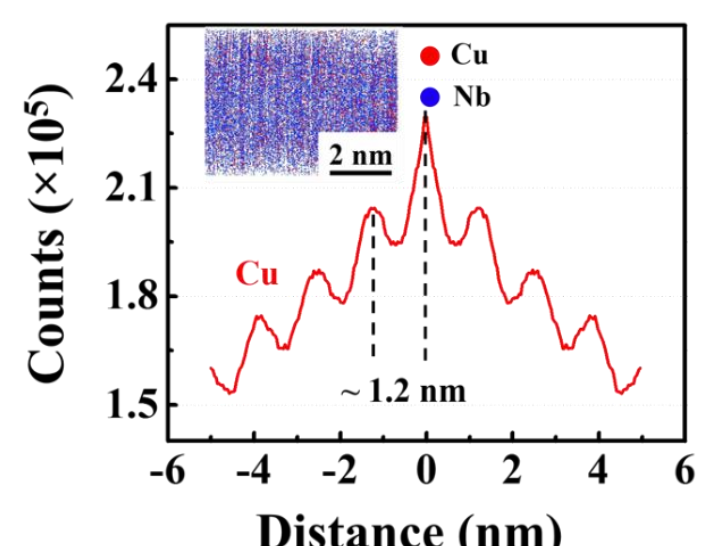

(b)

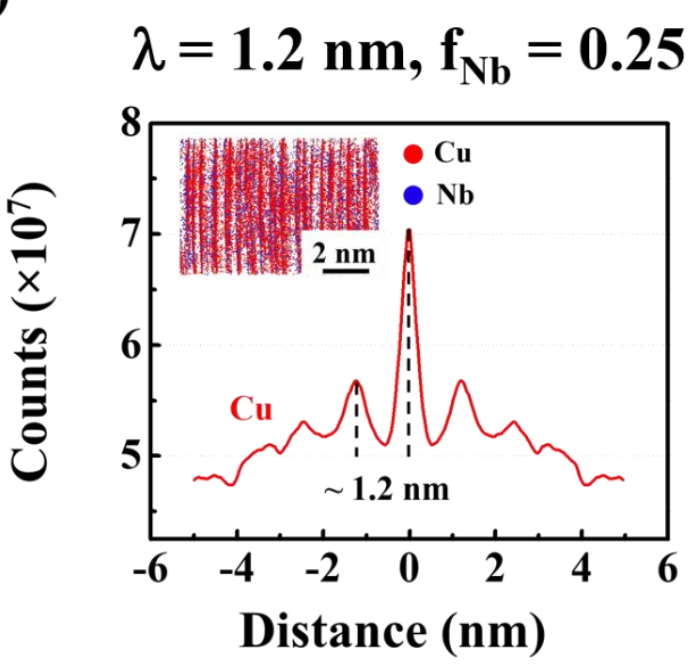

(d)

$$
\begin{aligned}
& \lambda=1 \mathrm{~nm}, \mathrm{f}_{\mathrm{Nb}}=0.5 \\
& \text { ROI Cu Clusters Nb Clusters }
\end{aligned}
$$
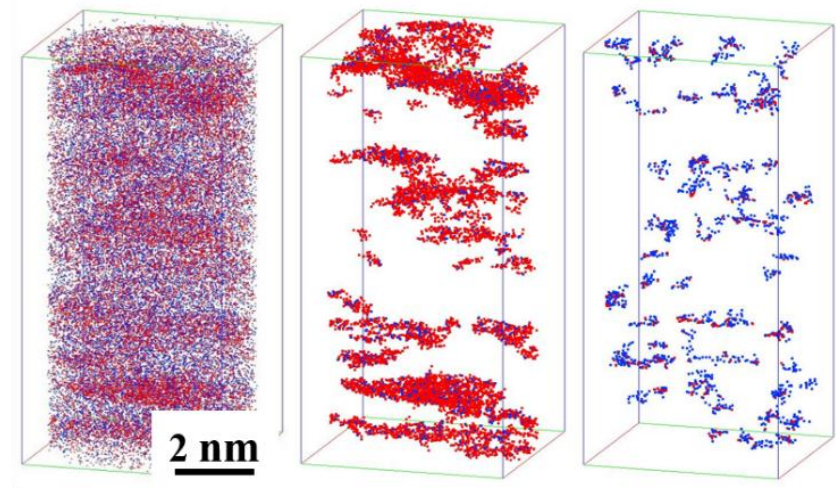

Fig. 9: (a) 1D concentration profile across the interfaces of the $\lambda=2 \mathrm{~nm} / \mathrm{f}_{N b}=0.5$ multilayer with atom ion map inserted. (b) Spatial distribution map of $\mathrm{Cu}$ atoms of $\lambda=1.2 \mathrm{~nm} / \mathrm{f}_{N b}=0.25$ multilayer with atom ion map inserted. (c) Spatial distribution map of $\mathrm{Cu}$ atoms of $\lambda=1.2 \mathrm{~nm} / \mathrm{f}_{N b}=0.75$ multilayer with atom ion map inserted. (d) Atom ion map of region of interest (ROI) and related $\mathrm{Cu}$ and $\mathrm{Nb}$ cluster maps of $\lambda=1 \mathrm{~nm} / \mathrm{f}_{N b}=0.5$ multilayer. Color available online. 


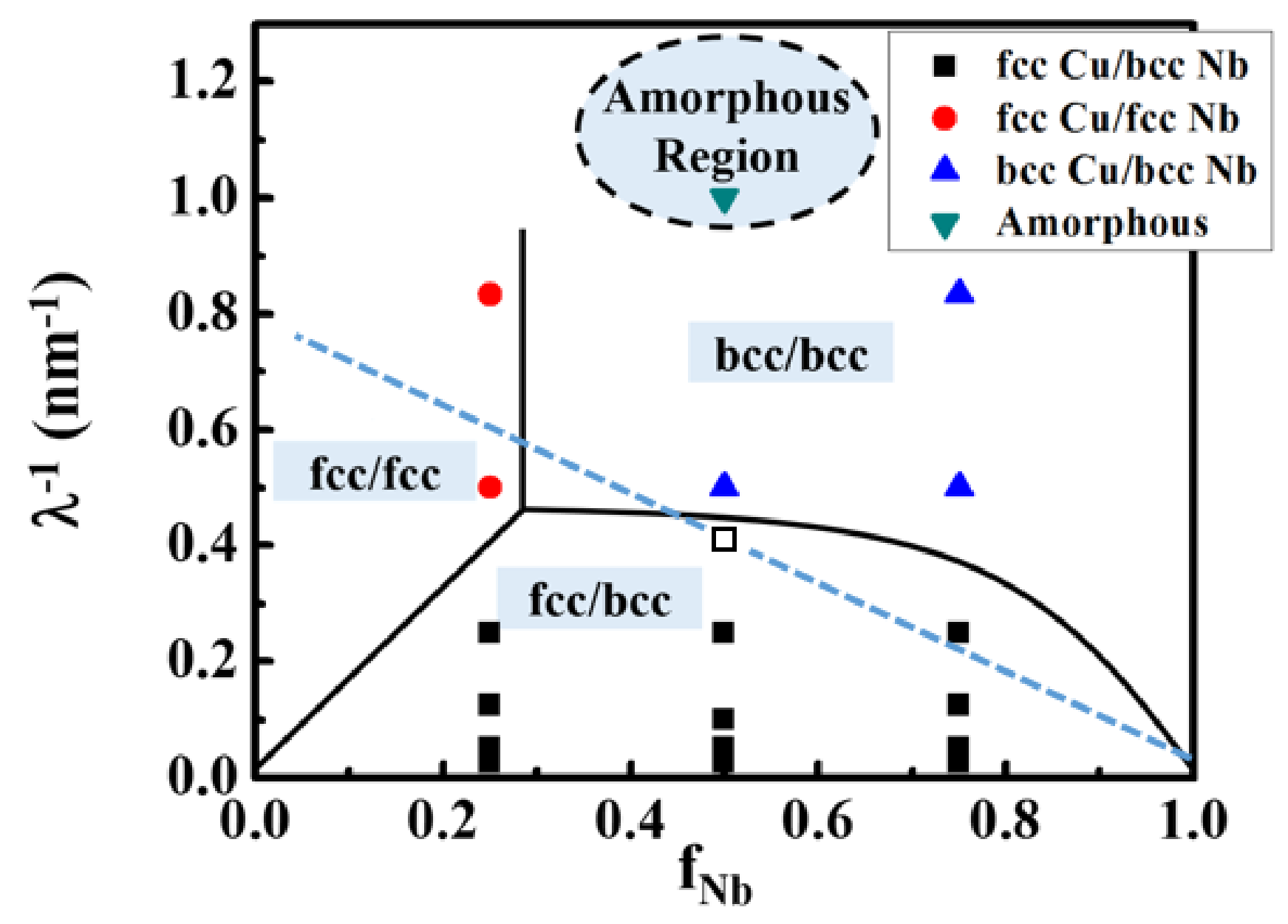

Fig. 10: Phase stability diagram. The dash lines represent the original predictions for phase space whereas the solid lines now represent the new experimental boundaries created by this report. Filled rectangular represents multilayers with fcc $\mathrm{Cu} / \mathrm{bcc} \mathrm{Nb}$ phases. Filled circle represents multilayers with $\mathrm{fcc} \mathrm{Cu} / \mathrm{fcc} \mathrm{Nb}$ phases. Up triangle represents multilayers with bcc $\mathrm{Cu} / \mathrm{bcc} \mathrm{Nb}$ phases. Down triangle represents multilayers with amorphous phase. Solid and dashed line represents phase boundary of different phases. 


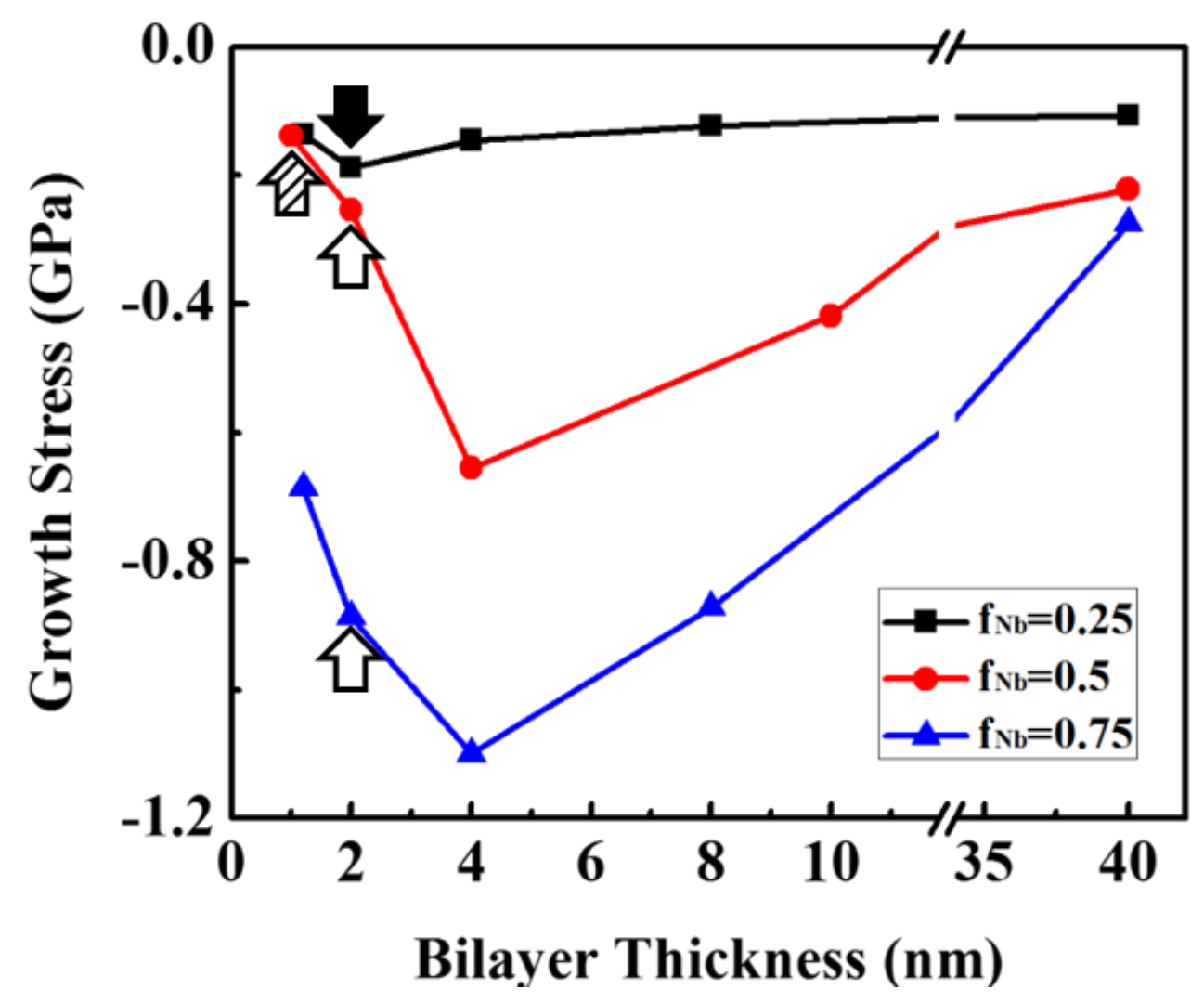

Fig. 11: Growth stress versus bilayer thickness curves for (a) $\mathrm{f}_{N b}=0.25$, (b) $\mathrm{f}_{N b}=0.5$ and (c) $\mathrm{f}_{N b}=0.75 \mathrm{Cu} / \mathrm{Nb}$ multilayers. Solid arrow represents the formation $\mathrm{fcc} \mathrm{Nb}$ phase. Hollow arrows represent the formation of bcc $\mathrm{Cu}$ phase. Slashed arrow represents the formation of $\mathrm{Cu} / \mathrm{Nb}$ amorphous phase. 


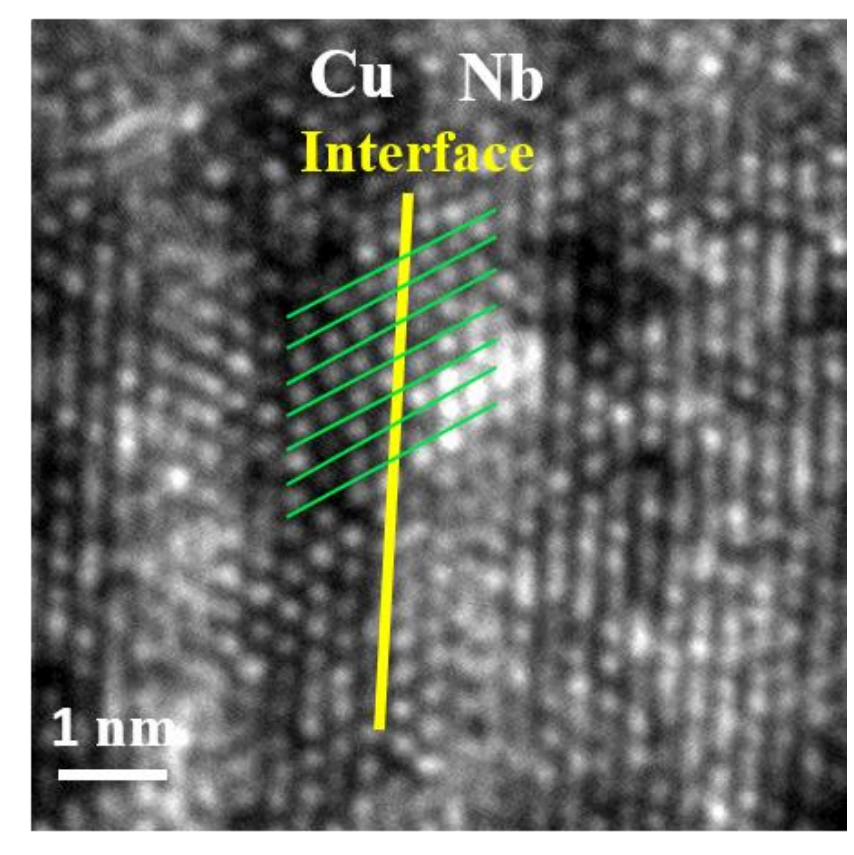

$$
\lambda=2 \mathrm{~nm}, \mathbf{f}_{\mathrm{Nb}}=\mathbf{0 . 5 0}
$$

Fig. 12: (a) HR-TEM cross-section image of $\lambda=2 \mathrm{~nm} / \mathrm{f}_{N b}=0.5$ multilayers at interface with the present of no-distortion coherent interfaces (indicated by green lines). Color available online. 
(a)

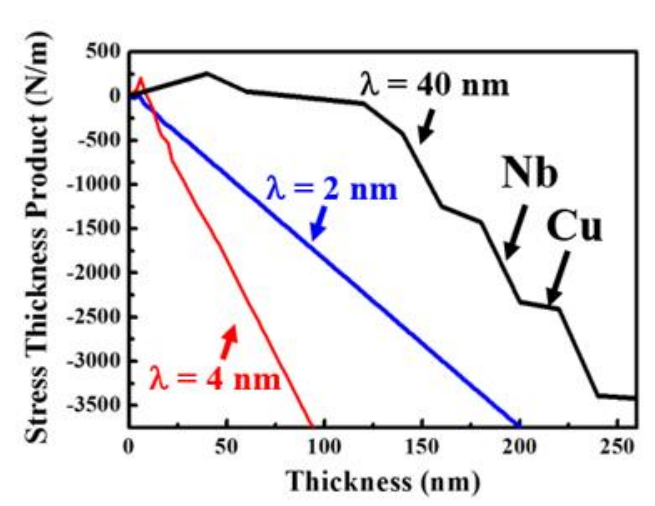

(c)

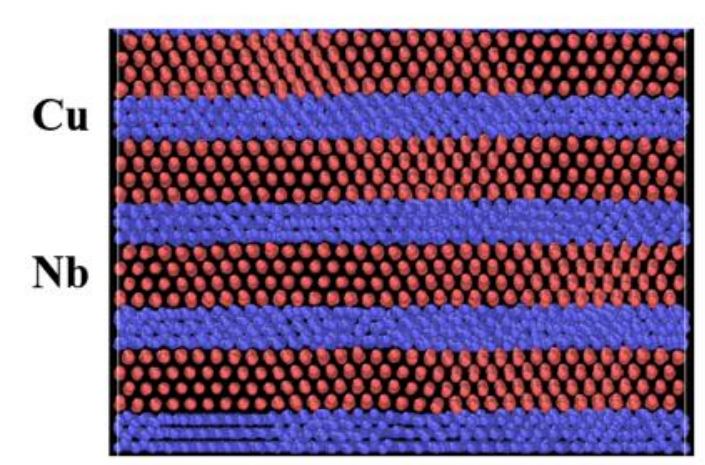

(b)
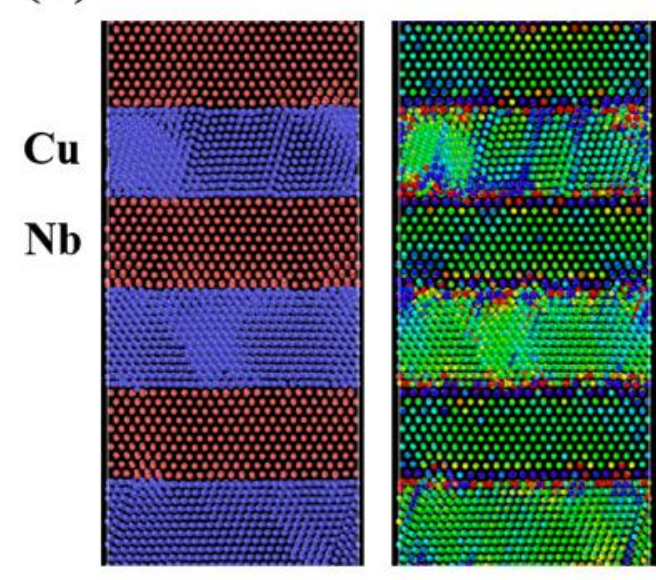

$\lambda=4 \mathrm{~nm}, \mathrm{f}_{\mathrm{Nb}}=0.5$

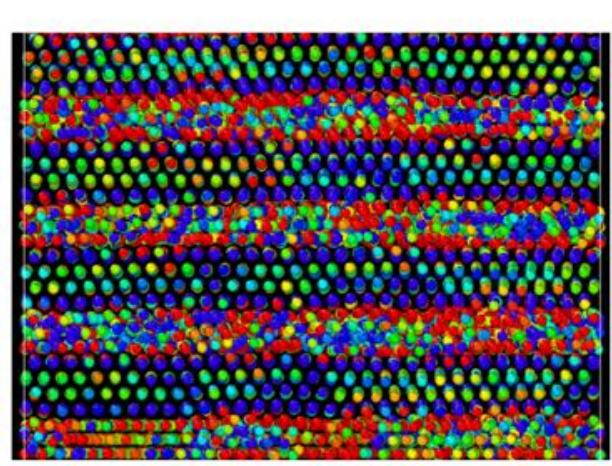

$-2.0 G P$

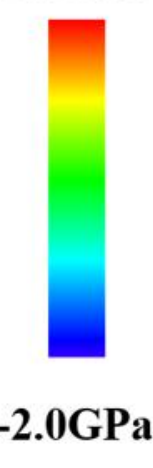

(d)

2.0GPa

Nb
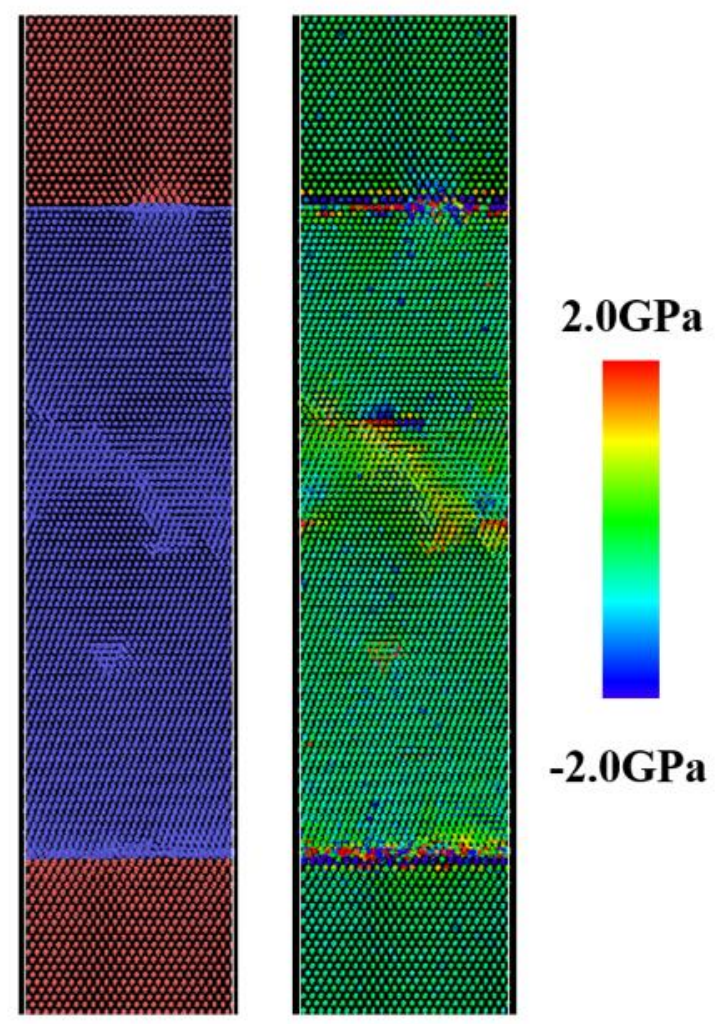

$\lambda=40 \mathrm{~nm}, \mathrm{f}_{\mathrm{Nb}}=0.5$

$\lambda=2 \mathrm{~nm}, \mathrm{f}_{\mathrm{Nb}}=0.5$

Fig. 13: MD simulation of $\mathrm{f}_{N b}=0.5$ for various bilayer spacings (a) Overall growth stress (b) atomic stress state distrubtion in fcc $\mathrm{Cu} / \mathrm{bcc} \mathrm{Nb}$ at $\lambda=4 \mathrm{~nm}$ (c) atomic stress state distrubtion in bcc $\mathrm{Cu} / \mathrm{bcc} \mathrm{Nb}$ at $\lambda=2 \mathrm{~nm}$ (d) atomic stress state distrubtion in $\mathrm{fcc} \mathrm{Cu} / \mathrm{bcc} \mathrm{Nb}$ at $\lambda=40 \mathrm{~nm}$. 
Fig. A1: Representative plan-view PED scans and inverse pole figures of labeled bilayers with corresponding grain size histograms under each scanned region.

$20 \mathrm{~nm} / 20 \mathrm{~nm} \mathrm{Cu} / \mathrm{Nb}$ multilayers
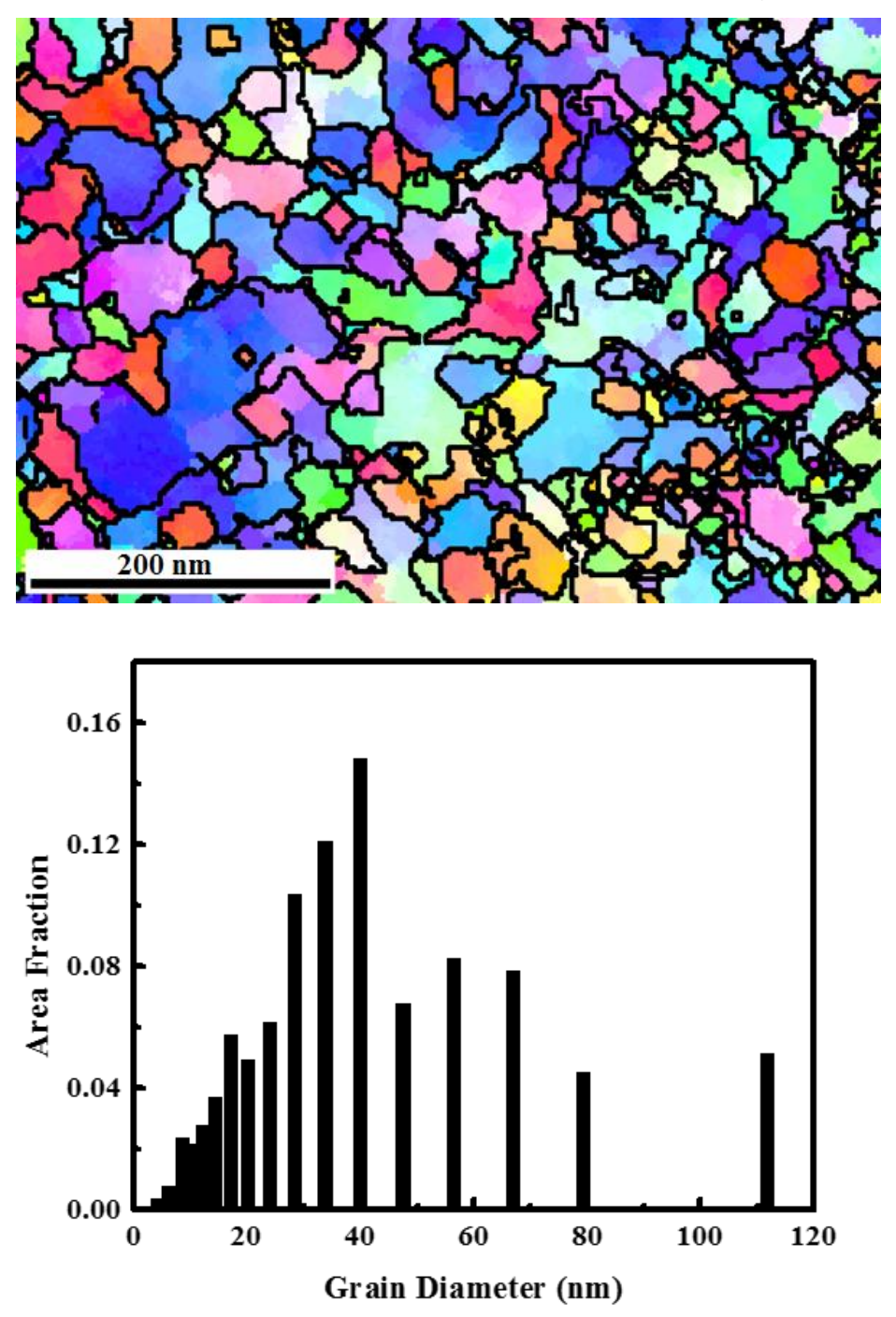

Average grain size $=\mathbf{4 0 . 7} \mathbf{n m}$
$2 \mathrm{~nm} / 2 \mathrm{~nm} \mathrm{Cu} / \mathrm{Nb}$ multilayers
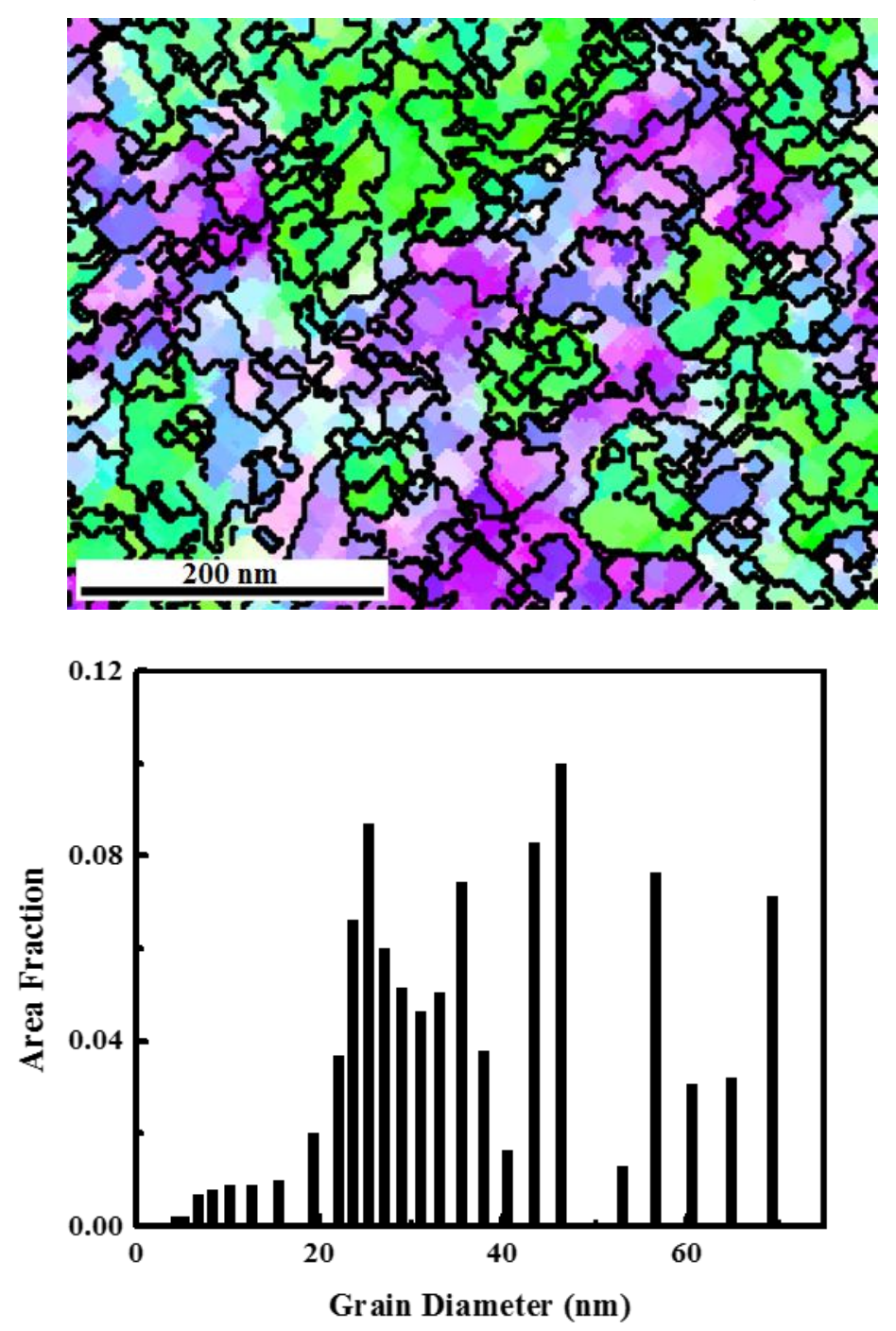

Average grain size $=\mathbf{3 8 . 5} \mathbf{n m}$
$1 \mathrm{~nm} / 1 \mathrm{~nm} \mathrm{Cu} / \mathrm{Nb}$ multilayers
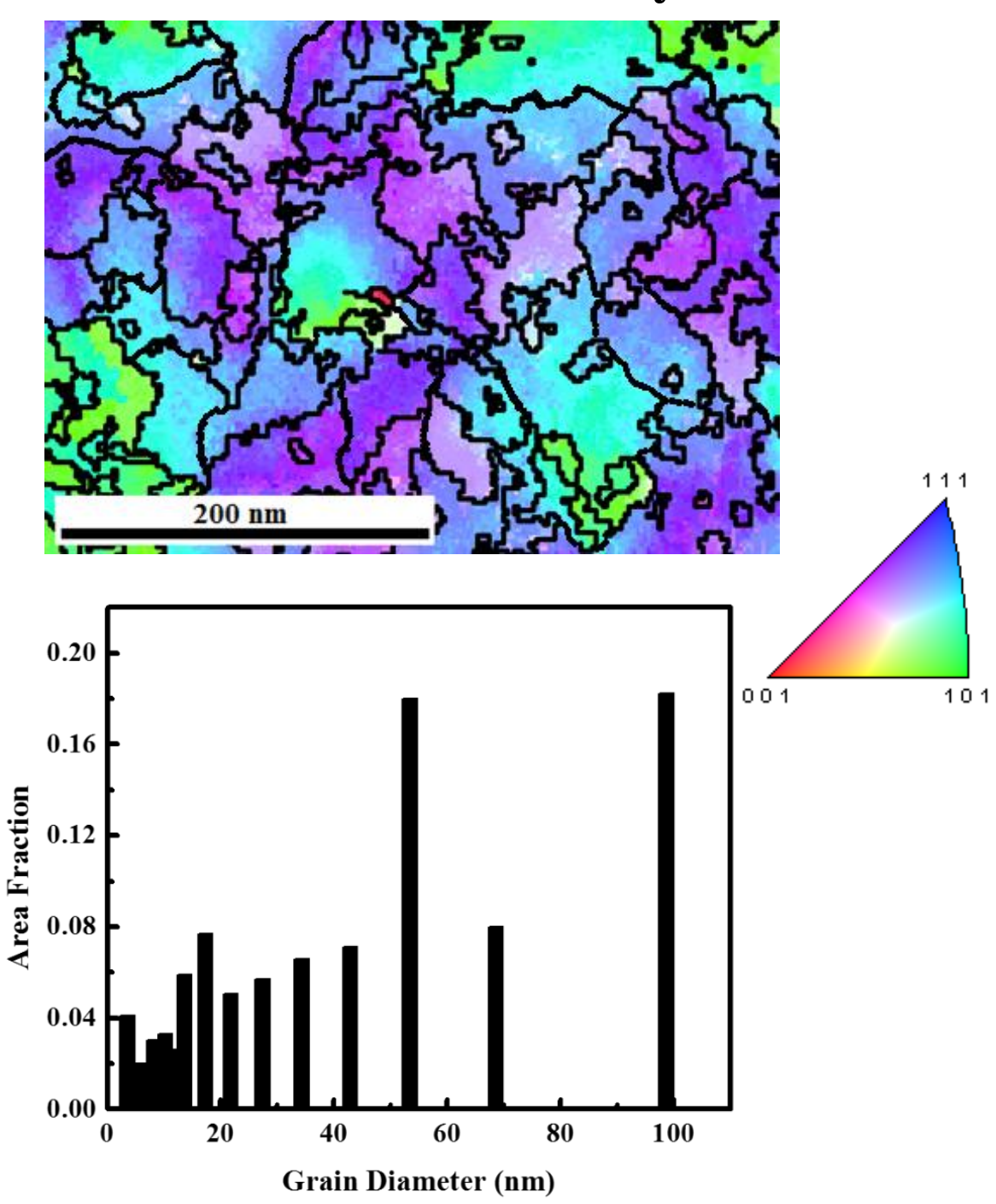

Average grain size $=\mathbf{4 4 . 5} \mathrm{nm}$ 
Fig. A2. A representative atom of $1 \mathrm{~nm} \mathrm{Cu} / 1 \mathrm{~nm} \mathrm{Nb}$ with its accompanying time-offlight mass spectrum. Note that no argon or oxygen was detected in the film. The presence of hydrogen is a known vacuum containment gas and believed not to have stabilized the phases evident by the diffraction results.

Mass Spectrum of $\mathrm{Cu} 1 \mathrm{~nm} / \mathrm{Nb} 1 \mathrm{~nm}$ Multilayers
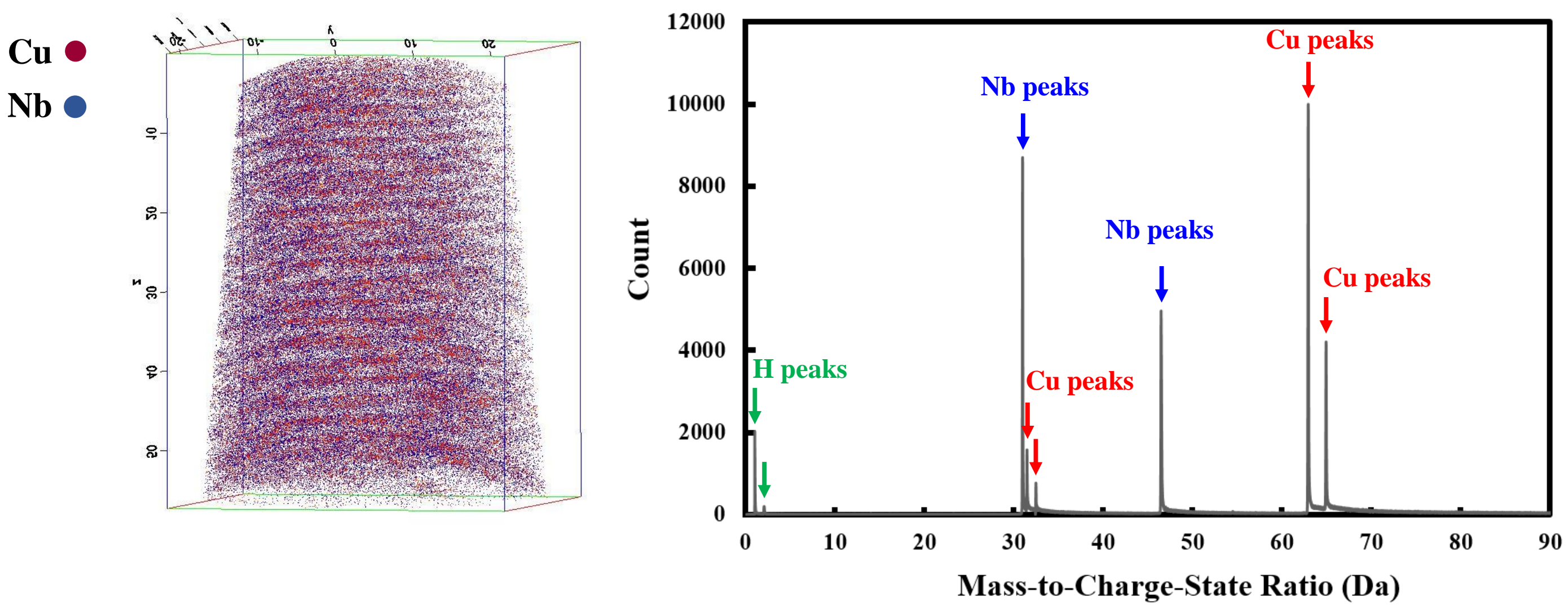


\begin{tabular}{|c|c|c|c|c|c|}
\hline $\begin{array}{l}\text { Nb } \\
\text { volume } \\
\text { fraction }\end{array}$ & $\begin{array}{l}\text { Bilayer } \\
\text { thickness } \\
(\mathbf{n m})\end{array}$ & $\begin{array}{l}\text { Phase ID } \\
\text { for } \mathrm{Cu} / \mathrm{Nb}\end{array}$ & $\begin{array}{l}\text { Experimental } \\
\text { interfacial } \\
\text { growth stress } \\
\text { per unit } \\
\text { length }(\mathrm{N} / \mathrm{m})\end{array}$ & $\begin{array}{l}\text { Calculated } \\
\text { overall growth } \\
\text { stress per unit } \\
\text { length }(\mathrm{N} / \mathrm{m})\end{array}$ & $\begin{array}{l}\text { Experimental } \\
\text { overall growth } \\
\text { stress per unit } \\
\text { length }(\mathrm{N} / \mathrm{m})\end{array}$ \\
\hline 0.25 & 40 & fcc/bcc & 13.5 & -334 & -257 \\
\hline 0.25 & 4 & fcc/bcc & -0.68 & -456 & -367 \\
\hline 0.25 & 2 & fcc/fcc & -0.70 & -372 & -370 \\
\hline 0.5 & 40 & fcc/bcc & -6.0 & -583.2 & -392 \\
\hline 0.5 & 4 & fcc/bcc & -7.3 & -982 & -1507 \\
\hline 0.5 & 2 & bcc/bcc & -2.0 & -620 & -625 \\
\hline 0.75 & 40 & fcc/bcc & -6.5 & -445 & -470 \\
\hline 0.75 & 4 & fcc/bcc & -20.3 & -1621 & -1858 \\
\hline 0.75 & 2 & bcc/bcc & -9.5 & -1439 & -1788 \\
\hline
\end{tabular}




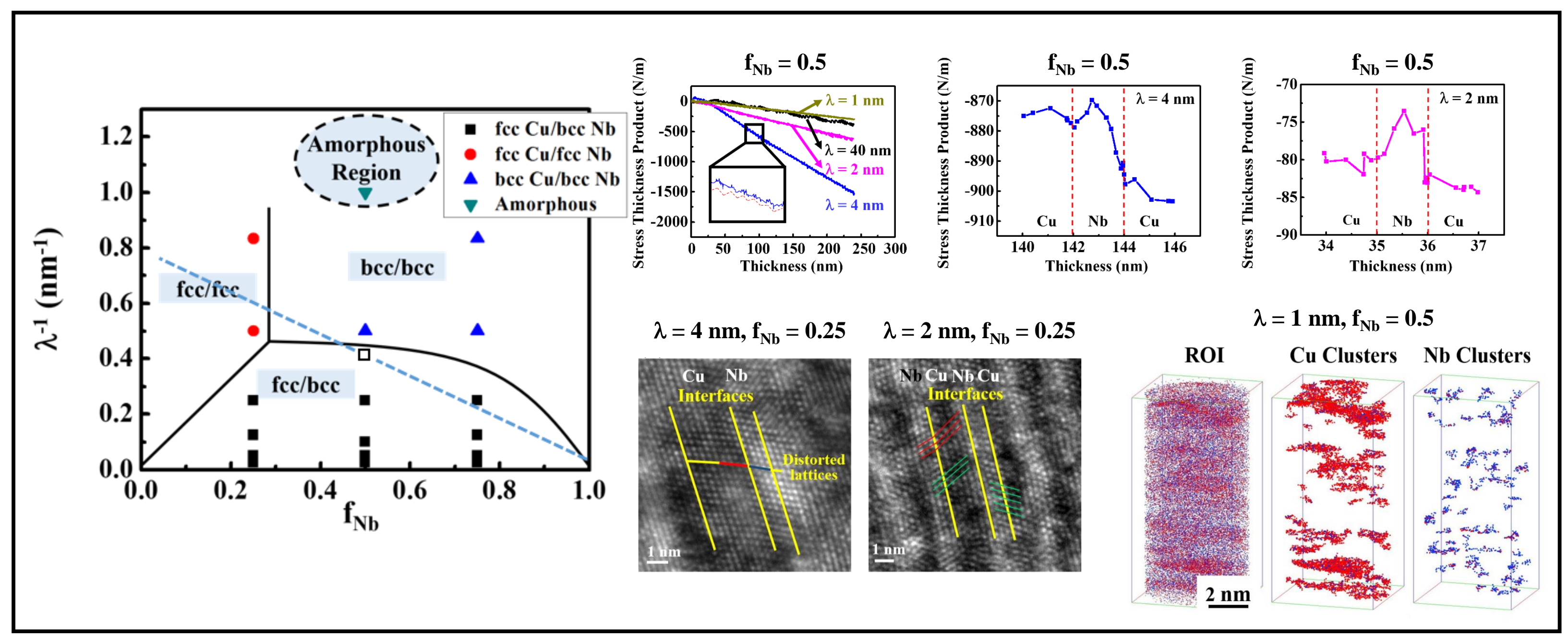

